(C2006 IEEE. Personal use of this material is permitted. However, permission to reprint/republish this material for advertising or promotional purposes or for creating new collective works for resale or redistribution to servers or lists, or to reuse any copyrighted component of this work in other works must be obtained from the IEEE. 


\title{
A Usability-Evaluation Metric Based on a Soft-Computing Approach
}

\author{
Elizabeth Chang, Member, IEEE, and Tharam S. Dillon, Fellow, IEEE
}

\begin{abstract}
Usability of software should measure both user preference and user performance. The notion of usability involves several dimensions. These dimensions include: system feedback, consistency, error prevention, performance/efficiency, user like/ dislike, and error recovery. Each of these dimensions are characterized by fuzzy aspects and linguistic terms. This paper develops a model for each of the dimensions using fuzzy-set theory. It then uses the Takagi-Sugeno fuzzy-inference approach for developing an overall measure of usability. Results are presented for several different user interfaces.
\end{abstract}

Index Terms-Fuzzy systems, usability, user interfaces.

\section{INTRODUCTION}

I $\mathrm{N}$ recent times, in industry and commerce, computers have moved from offline data processing or large-scale numerical calculation, to the online task of control, monitoring, and manipulation of individual processes. This change in the role of computers within industry has also been accompanied by a change in the types of people who seek to use them. The current groups of users have inherently little interest in the inner workings of the computer, nor do they wish to devote much time or effort to learning how to use them. This has resulted in a shift in the character of computer users, from those who are extremely knowledgeable about computers, to ones who are more strongly engaged in other activities such as managing, monitoring a process, quality control, or administration. The computer is merely a convenient tool, helping people solve their problems. The users, therefore, have little patience with mastering a particular operating system or command language, or an inflexible editor. They expect the computer to interact with them in a familiar language, and with an ease of manipulation, which allows them to devote their mental efforts to solving their own problem, rather than learning and remembering a set of cryptic commands to which the computer responds.

There are many examples of software systems that have not gained wide acceptance among users, and have been consigned to oblivion, or have had scant use, in spite of great expenditure on their design and implementation. There are other examples

Manuscript received December 13, 2002; revised October 7, 2003. This paper was recommended by Associate Editor S. Guerlain.

E. Chang is with the School of Information Systems, Curtin University of Technology, Perth W.A. 6845, Australia (e-mail: change @ cbs.curtin.edu.au).

T. S. Dillon is with the Faculty of Information Technology, University of Technology Sydney (UTS), Sydney N.S.W. 2007, Australia (e-mail: tharam@ it.uts.edu.au).

Digital Object Identifier 10.1109/TSMCA.2005.851349 of software that have been modified and updated many times. and are still not accepted by the user. An important reason for some of these failures is the inability to respond to the varied needs of the human user in the industrial and commercial context, and the difficulty of adapting general layouts to particular applications, as well as providing a suitable user interface (UI). The urgency of providing more suitable software has led to an upsurge of research activity into the fundamental needs of users. and the interaction of human operators with a computer. Thus, software houses and users of networked computer systems have commissioned theoreticians in such diverse fields as logical factor analysis, psychology, cognition, semantics, ergonomics, and physiology, to provide them with the methodologies they need to design good human-computer interfaces [1], [6], [8], [11], [17].

When one is carrying out the development of the UI utilizing a particular design methodology, it is important to realize that one cannot easily characterize or evaluate the impact a particular UI is likely to have on a given set of users. The field of evaluation of a UI otherwise known as usability evaluation, has become increasingly important with computer users, as explained above, being drawn from varied groups, from computer specialists to the average person in the street.

The usability of a computer software "is measured by how easily and how effectively it can be used by a specific set of users, given particular kinds of support, to carry out a defined set of tasks, in a defined set of environments" [15]. In the literature, a variety of approaches have been adopted in order to evaluate usability, and these can be categorized as follows: 1) empirical testing [10]; 2) inspection [12];3) comparative usability measures [5]; 4) formal complexity-based measures [18]; and 5) the metrics for usability standards in computing (MUSIC) methodology [2].

Empirical testing essentially involves testing the software product in a laboratory environment, which seeks to simulate as closely as possible the conditions under which the user uses it in the field. The user is asked to carry out designated tasks using the software product. The user's interactions with the system are recorded, using video equipment and audio equipment, sometimes with timing markers. One can also use a questionnaire. The primary focus of empirical testing is to uncover problems that the user is experiencing with the product. If the product is still in a prototype stage, then the results of this testing can be utilized for refining the final version of the product. However, if the product is in the final stage of development, this approach is useful for determining whether one selects a given product. Three different testing laboratory setups can be utilized for empirical testing and these include: 
1) fixed laboratories; 2) portable laboratories; and 3) remote laboratories.

The main advantage of empirical testing is that it is able to directly monitor the performance reactions of the user to the particular interface, and one does not rely on a usability expert trying to second-guess what a user's reaction might be. The disadvantages are that it is only useful for testing an implemented product and, therefore, the ability to make changes to correct the problem immediately is greatly reduced. A further disadvantage is the fact that it can focus on and identify specific problems that the user experiences with a given set of tasks, but does not give measures of the quality of the UI, either along specific dimensions or an overall figure of merit. It is also very time consuming, requiring tedious reviews of audio and video recordings. A new approach using automated usability testing that overcomes these weaknesses has been developed by Chang and Dillon [4].

The second major approach of usability evaluation is the use of usability-inspection methods. Usability-inspection methods essentially consist of a group of experts studying the design layout of a UI. They provide a set of comments on various aspects of the UI based upon their expert opinion. These comments are then utilized to alter various aspects of the design of the UI so that it overcomes the problems that are identified. Since the usability expert does not rely on a usabilitytesting laboratory, the costs associated with usability-inspection methods are much lower. Two of the most widely used usability-inspection methods are: 1) cognitive walkthrough and 2) heuristic evaluation. The difficulties here are twofold, namely: 1) they are highly dependent on the ability of the usability expert and 2) they are based on the assumption that the assessments made by the expert map onto the experiences and opinions of the user. Further, this type of approach is again more useful for identifying particular defects in the interface rather than determining figures of merit of the overall interface or along particular dimensions.

Comparative usability measurement utilizes a series of subjective and objective measures as the basis for comparing the usability of a given group or range of products. The objective measures essentially relate to user performance, and the subjective measures relate to user satisfaction. Comparative usability measurement begins with a task analysis to identify a set of tasks that are to be utilized in a test by the test subjects. Each product is then tested a number of times, and on each occasion, it is tested by different users. The test subjects also have to complete a scoresheet as they carry out each task and they also have to fill out a satisfaction questionnaire. The satisfaction questionnaire [19] shows assessors a number of factors relating to user satisfaction. The objective or performance-related measures that are utilized include the time required to complete each task, the time and frequency of help references, and the number of successfully completed parts of the tasks, and these are turned into productivity measures. Essentially, these measures are currently frequently employed on completed products and they are not utilized during development.

The class of complexity measures is applied during the design stage. They essentially analyze the design and develop mathematically based measures that are supposed to capture the complexity of the design of the UI. A good description of these complexity measures is given in Thimbleby [18], where he outlines a series of models that are utilized for calculating the complexity under a variety of different situations. Complexity measures, however, have a number of serious limitations. There is little experimental evidence to support the belief that these complexity measures are effectively related to user performance and user preference. For instance, the suggestion that machine learning, which provides algorithms to learn finite-state machines, is a useful model of the user learning by trial and error, is a highly tenuous connection.

MUSIC [2] is a methodology developed as the result of an Esprit project in Europe. The MUSIC methodology has four major aspects: 1) usability context and analysis; 2) user performance measurement; 3) user satisfaction measurement; and 4) cognitive-workload measurement.

Usability-context analysis is essentially concerned with examining the equipment (hardware and software) and the types of users and their experience, the type of task, including the breakdown of its frequency and duration, and the environment itself, including the organizational aspects, structure, including job design, and work-related conditions. User performance measurement is carried out using a number of submeasures, and these are: task effectiveness, efficiency, productive period, and relative user efficiency. User satisfaction measures are obtained by using the software usability measurement inventory. The last group of measures that MUSIC provides are the cognitiveworkload measures, and these essentially evaluate the mental effect required by users to complete a task. There are two different approaches utilized and these are: 1) the subjective mental-effort questionnaire and 2) the task loading index.

While the MUSIC methodology is a good attempt at obtaining measures, it can be improved in three areas.

1) Its focus is on measuring aspects of the user in relation to the interface such as user satisfaction and user performance. It does not measure aspects of the interface that might influence the user's interaction with it. This second set of measures will be useful in helping determine broad areas of improvement.

2) Several of the aspects are characterized by fuzzy and linguistic terms, and the MUSIC methodology does not properly represent these.

3) Lastly, it does not provide a single overall measure of usability. It does not attempt to combine its four dimensions to obtain a single overall measure of usability. An important aspect of the comparative usability-measurement techniques above and the MUSIC methodology discussed above, is that each of them is used in conjunction with an implemented product, rather than during the design stage. The advantage of utilizing the measurement methodology in the implementation stage is that it allows direct user interaction with the interface and, therefore, gives a more reliable index of user satisfaction and user performance, as well as the cognitive workload associated with the particular interface. The disadvantage of it is that, since it is utilized in the implementation stage, it does not allow easy alteration of the UI, such as would occur if 
the methodology were to be used during the design and development stage. However, evaluating the usability at the design stage could result in unreliable measures.

In this paper, we will develop an approach that characterizes key aspects of the UI and key aspects of the user in relation to the interface. Further, each of these dimensions will be represented within a fuzzy framework, and measures along each dimension are developed. In addition, an overall figure of merit will be developed for the interface.

\section{Problem Definition}

Usability evaluation has two particular objectives: 1) to isolate specific problems with the UI and propose methods for correcting these and 2) to obtain figures of merit of the usability for a UI to allow comparisons between either different implementations, or different designs of a UI. In this paper, we will concentrate largely on the problem of obtaining the figures of merit of the UI, both for all the different aspects of the UI, as well as giving an overall figure of merit. Usability itself is a difficult concept to quantify. It has several dimensions and several factors seem to impact upon it. However, putting these dimensions and factors together to obtain a figure of merit of usability is a very difficult issue, because each of them involves linguistic terms and fuzzy concepts.

Previous studies [2], [5], [7], [10] determined that many factors go into making user performance and user preference, and these influence the usability of the software. So when one examines each of these, one notices that there are several factors that comprise the characteristics of usability. In this paper, the problem we intend to address is to isolate the most important of these factors and work out a means of characterizing each of them individually, and then to develop a technique that seeks to combine them in such way that we obtain a composite overall figure of merit for the total UI. The difficulty in combining these factors lies in the fact that each one of these factors, on its own, is an imprecisely defined concept and has fuzzy aspects. Furthermore, the notion of usability itself cannot be readily quantified into a numerical value, which can be immediately understood as a figure of merit. The notion of usability itself has a number of fuzzy aspects. For these reasons, we have chosen to examine fuzzy-systems theory as a mechanism for characterizing each of these individual factors and dimensions and also for characterizing usability itself.

Furthermore, we will also define an overall methodology that allows one to systematically utilize this theoretical development of a fuzzy-system-based usability measure in such way that one progressively utilizes aspects of it in the design, prototyping, and implementation stages to improve the UI.

\section{A. Modeling Factors That Impact Usability}

Previous researchers [2], [10], [13] have identified a number of factors that contribute to usability. These factors influence both the user's performance and whether or not the user likes or dislikes the interface (namely, user acceptance).
A perusal of the literature helps us identify the factors, discussed below, that have to be taken into account. Thus, we note that system feedback by the system, to keep the user informed about the state of the system and/or the point in the task that is being carried out, is very important. This must provide relevant and necessary messages that prompt the user and provide advice as to why certain actions cannot be carried out, etc. It is also related to the clarity of the messages. System Feedback will affect user performance, as well as user acceptance of the interface.

Another factor of considerable importance is consistency. The UI should be consistent from screen to screen throughout the whole application, both in placement of actions and messages and color use, etc. It should also be consistent, where possible, with other applications that it might be utilizing or interoperating with. Preferably, there should be consistency with other applications in the domain as well. This will have the effect of reducing the mental effort involved in switching between screens or applications, and also reduce the uncertainty the user faces.

Two other factors that influence performance are error prevention and error recovery. Error prevention aims to ensure that the design of the UI is such that it helps the user to avoid making errors, while error recovery relates to the ability of the user to recover once the user is in an error state. Both of these factors influence the performance of the user, as well as the acceptance of the UI by the user.

In addition to these factors, we need to measure the performance of the user in carrying out a certain number of typical tasks. The reason we need to measure this directly is that there could be many other factors, some of which may be undifferentiated, which go into the overall performance that a user exhibits with a particular UI. Hence, it is necessary to carry out direct measurements of performance.

In addition to performance, an important element of user responsiveness is the user's like or dislike of a particular UI. Again, many factors go into making up this aspect of the user's response, some of which may not be totally understood or differentiated. Hence, it is necessary to have a measurement of user like/dislike.

It is important to realize that the first four factors considered, namely system feedback, consistency, error prevention, and error recovery, impact on performance and user like/dislike and, therefore, are not necessarily independent of the last two. However, there may be other undifferentiated elements also present in the last two.

Therefore, in order to characterize usability of UIs, we will employ the following factors: 1) system feedback; 2) consistency; 3) error prevention; 4) performance/efficiency; 5) user like/dislike; and 6) error recovery.

These factors have also been identified by previous researchers as contributing to usability [2], [10]. However, with each factor, we need a method of determining the quality of the UI with respect to it. We discuss each of these in turn to work out how they will be measured.

In attempting to characterize these quantities, we need to first of all measure quantities directly related to these factors. In determining what to measure, it is important that, as we 
are trying to evaluate usability, there is a direct relationship between the user's interaction with the software, rather than an evaluation with a third party. Hence, we decided to set up a testing procedure to carry out the measurements required as explained in Section IV.

These measurements involved an observer, the user, and the use of software to carry out a designated series of tasks. In the rest of this section, we will take each of these factors in turn and show how a fuzzy representation of them can be developed.

I) System Feedback: The system should always provide users with the appropriate feedback, so that they can be informed about what is going on in the system at any time.

System feedback is characterized by several aspects, including the following.

1) Is there error localization?

2) If an action is not permitted, does the system say why?

3) Does the system give prompts as to what to do next?

4) Does the system let one know where one is?

5) Does the system tell one why one cannot do something?

In order to directly measure the users' interaction with the software, in relation to these aspects, the test was set up as explained above and in Section IV. The number of times that the system displayed inadequate feedback in this interaction of the user with the software was then measured during the test.

Inadequate system feedback has several components, and these include:

1) number of times dialogue/feedback is missing;

2) number of times dialogue/feedback is unnecessary;

3) number of times system feedback confuses the user;

4) number of messages that are irrelevant;

5) number of actions taken that lead to a repeat of the same system feedback message; and

6) number of times the user makes the wrong choice of action based on system feedback.

Each time any one of these is not satisfied, we can say that on this occasion, system feedback was inadequate. Thus, we will count this as contributing one unit to a measure called "count of number of times of inadequate system feedback." It could be argued that not all of these components are equally severe occasions of inadequate system feedback, and perhaps some incidents should be given higher weighting than others. The current state-of-the-art of the study of usability has yet to make distinctions between the severity of these different components. In the absence of such information, we give each of these the same weight. This does not preclude the fact that in future, these components could be grouped together on the basis of advice from a usability expert and each group given a different weight. However, while the count obtained for a particular piece of software would be different, it does not affect the basic methodology proposed in the paper. For similar reasons, the counts will be weighted by one in measurements of all the subsequent factors. System feedback itself is usually characterized using linguistic terms such as Good, Average, Poor. The fuzzy-term set, therefore, is [Good, Average, Poor\}. If we employ the generalized Bell (G-Bell) shapes for the membership functions, the fuzzy-membership feedback will look like those shown in Fig. 1.

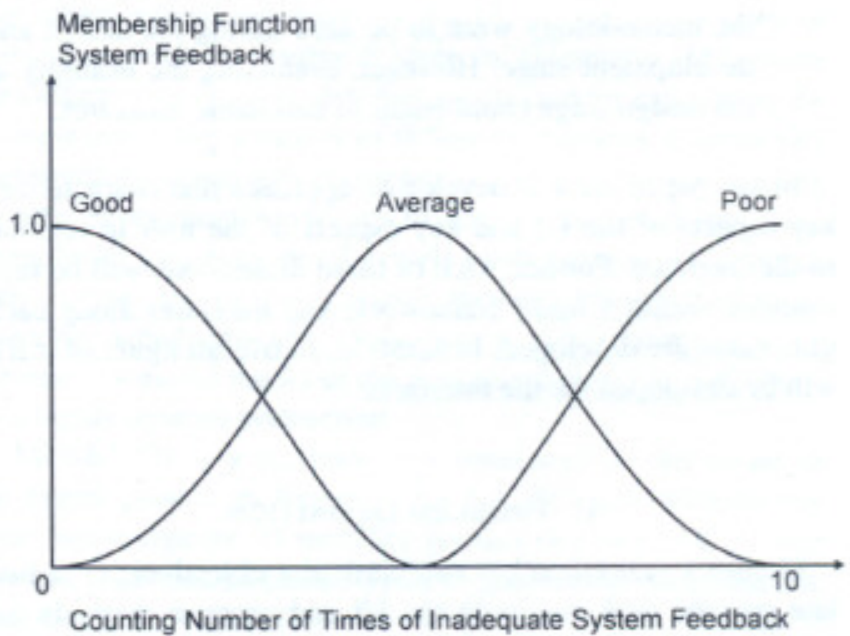

Fig. 1. Membership function for system feedback.

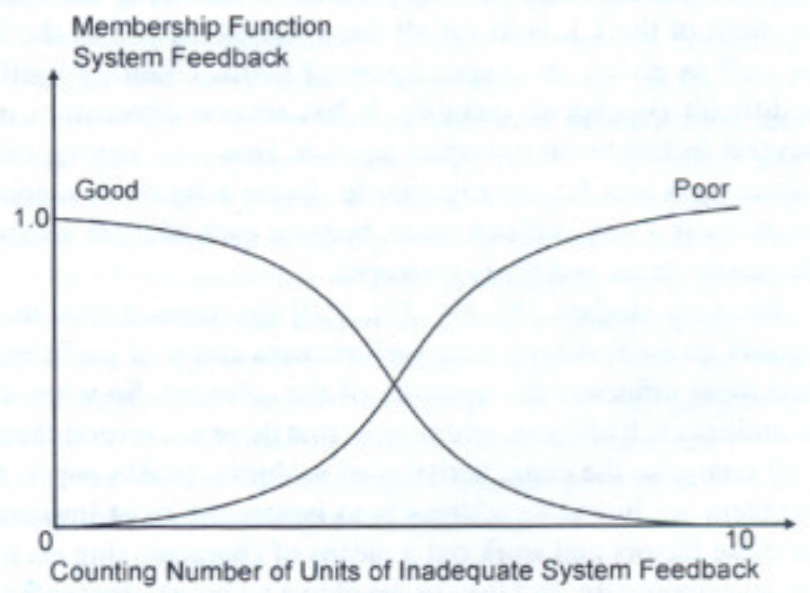

Fig. 2. Membership function for system feedback-two-fuzzy-set model.

Note that in Fig. 1, we have divided the universe of discourse (UoD) for system feedback into three domains corresponding to three different fuzzy sets, namely: Good, Average, and Poor. However, it is also possible to divide the UoD into two fuzzy sets as shown in Fig. 2. We will study both the fuzzy sets shown in Figs. 1 and 2, and compare results using these for modeling each of the input factors. The reasons for studying both the two-fuzzy-set case and the three-fuzzy-set case are as follows: the amount of computational effort required in the two-fuzzy-set case is considerably lower; hence, it would be useful to determine the gain in usability evaluation obtained by employing the three-fuzzy-set case, so as to provide guidance to practitioners and future researchers as to the appropriate model to adopt.

To avoid repetition, the two-fuzzy-set partition of the UoD will not be repeated for the remaining factors, but it is to be understood that such a partition will also be studied for the factors, consistency, error prevention, performance, error recovery, and user like/dislike. 


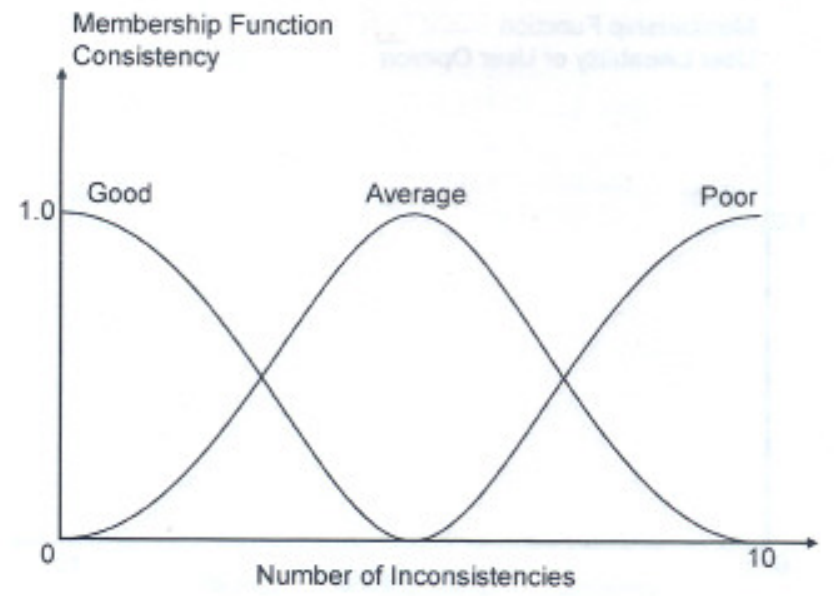

Fig. 3. Membership function for consistency.

2) Consistency: The look, feel, and behavior of the interface should be consistent throughout the application and also be consistent with other applications in the same domain. Most guidelines seek to bring this about. This consistency should be across a variety of issues such as message-display methods, color use, key definition, data-entry methods, etc.

If the UI is consistent, it reduces the amount of uncertainty that the user faces when using the interface. It also is likely to reduce the number of erroneous interpretations or actions that the user makes. As was the case with system feedback, we characterize inconsistency in the interface by utilizing a count of the number of inconsistencies identified. Consistency of the interface has a number of components and these include consistency with the respect to the following:

1) message-display methods (prompts, warnings, helps);

2) color use (entry form, foreground/background, menu, and submenu);

3) keys definition;

4) data-entry method;

5) menu, dialogue, and window-display methods;

6) menu hierarchy that is consistent with the real world;

7) terminology used is the same as in real life in that do main; and

8) menu options have to be consistent with menu title.

Each time anyone of these is not satisfied between screen-toscreen displays, we will count this as contributing one unit to a measure called inconsistency. The fuzzy-term set is [Good, Average, Poor $\}$ and the fuzzy membership for consistency is shown in Fig. 3.

3) Error Prevention: Error prevention is an important goal of the design of the UI. If the UI specifically helps the user to avoid making errors, it increases his efficiency. It will also reduce the level of frustration the user is likely to experience with the UI and, therefore, bring about greater acceptance of the UI by the user. There are several aspects that need to be taken into account in measuring error prevention, and these include the following:

1) number of errors encountered during task;

2) number of wrong key strokes/press causing error messages;

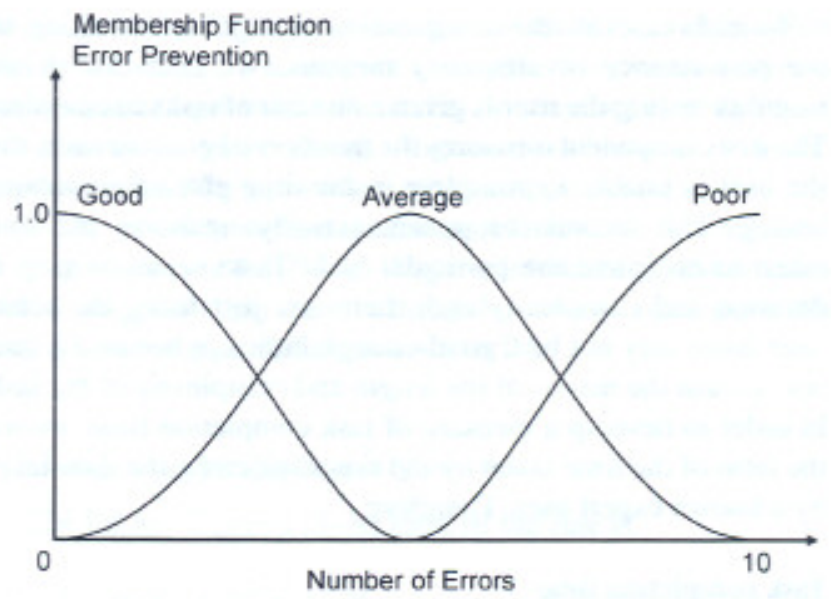

Fig. 4. Membership function for error prevention.

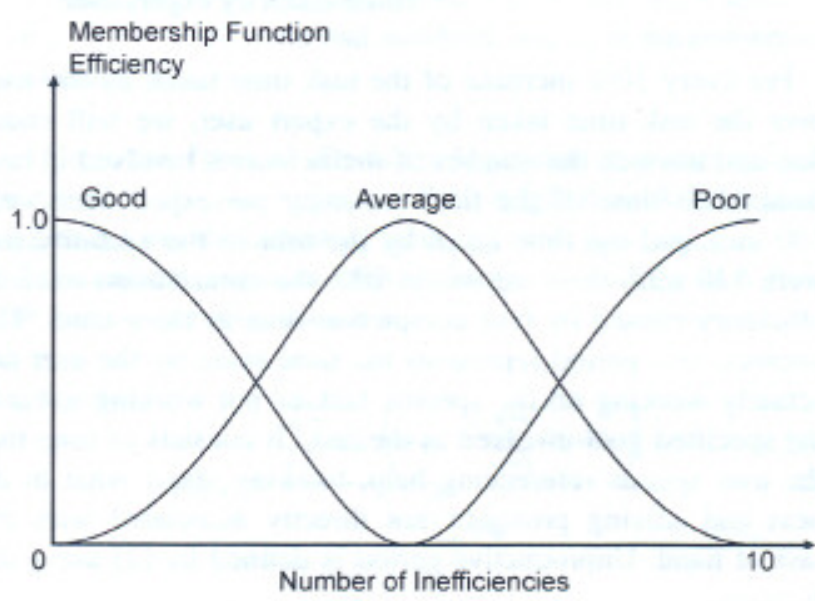

Fig. 5. Membership function for efficiency.

3) number of times the same key is pressed without the desired response;

4) number of extra key presses that are unnecessary;

5) number of times the same error is encountered; and

6) number of steps missing compared with real world execution.

Each time any one of these is not satisfied, we count one unit towards error occurrence. Each error encountered represents an occasion when error prevention has not been met. The fuzzyterm set is [Good, Average, Poor\}. The fuzzy membership for error prevention is shown in Fig. 4.

4) Performance/Efficiency: Performance or efficiency is a quality of the UI, which characterizes how effectively or efficiently the user can complete his tasks. Performance and efficiency have a number of components, and these are as follows:

1) number of goals/tasks not achieved;

2) time taken for task completion;

3) unproductive period; and

4) percentage of task not completed.

Each time any one of these is not satisfied, we count one unit towards inefficiency. The fuzzy-term set is \{Good, Average, Poor \}. The fuzzy membership function is shown in Fig. 5. 
To elaborate on the components that go into making up the performance or efficiency measure, we note that during usability testing the user is given a number of tasks to complete. The first component measures the numbers of goals or tasks that the user is unable to complete in the time given for usability testing. The second component actually measures the time taken to complete the particular task. Tasks seem to vary in duration and complexity and, therefore, just using the actual time taken may not be a good-enough indicator because it does not contain the notion of the length and complexity of the task. In order to develop a measure of task completion time, we use the ratio of the time taken by the user divided by the time taken by a known expert user. Therefore

Task completion time

$$
=\frac{\text { time taken by user to complete task }}{\text { time taken by expert user }} .
$$

For every $10 \%$ increase of the task time taken by the user over the task time taken by the expert user, we will count one unit towards the number of inefficiencies involved in task completion time. If the time taken by the expert user were $100 \mathrm{~min}$, and the time taken by the user in the usability test were $130 \mathrm{~min}$, then we would take the contribution towards efficiency caused by task completion time as three units. The unproductive period represents the time spent by the user not actually working on the specific task or not working towards the specified goal involved in the task. It consists of time that the user spends referencing help, thinking about what to do next and solving problems not directly associated with the task at hand. Unproductive period is defined by [2] using the formula

\section{Unproductive period}

$$
=\text { help time }+ \text { search time }+ \text { snag time. }
$$

The last component consists of the percentage of tasks the user did not actually complete, and it is calculated directly from the first measure of goals not achieved, and is given by

Percentage of task not completed

$$
=\frac{\text { goals not achieved }}{\text { number of goals expected to be achieved }} .
$$

5) User Like/Dislike or User Opinion: Unlike the previous measure, which measures the manner in which the UI facilitates user effectiveness or efficiency, the like/dislike factor measures user preference. This essentially indicates the level of satisfaction that the user feels with the system and the UI. There are, in fact, two alternative approaches that can be used to measure this user like/dislike.

\section{Approach 1:}

1) Count the number of times the user gives positive comments when testing the system.

2) Count the number of times the user grumbles or gives negative comments.

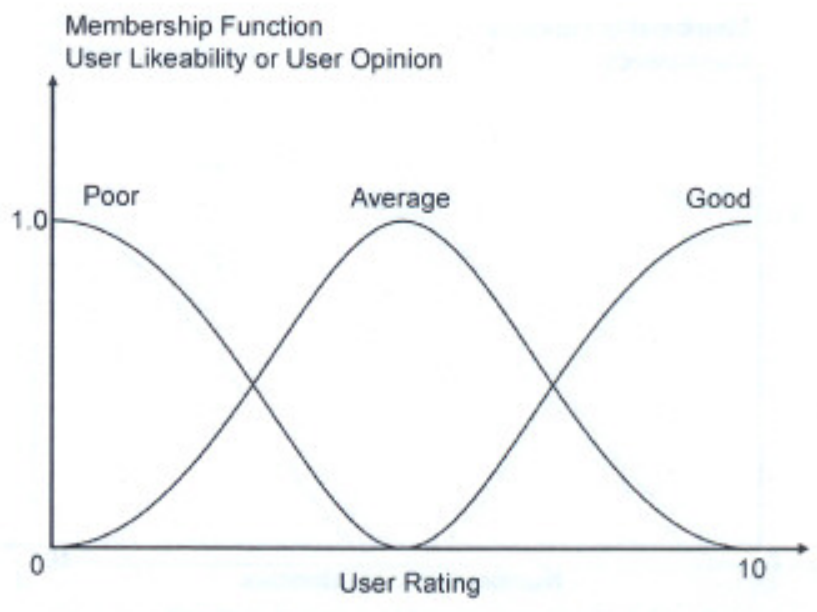

Fig. 6. Membership function for user likeability.

Approach 2: Ask the user to indicate on a scale of 1 to 10 the likeability of the system. From approach 1, we add one unit to user likes for 1) or we subtract one unit for 2). In approach 2 , we use a scale of 1 to 10 and convert it using the membership function into the fuzzy sets. The fuzzy-term set, therefore, is \{Poor, Average, Good\}. The fuzzy membership function is shown in Fig. 6.

6) Error Recovery: Error recovery is the quality of the system of the UI that allows the user to exit from a situation that the user did not intend to be in. Users frequently choose the wrong option or enter the wrong data and they are likely to find themselves in an error state, from which they need to recover. The manner in which the system facilitates this error recovery could reduce the time the user spends recovering from this error state. Error recovery consists of a number of components, and these include:

1) number of times the user has to redo the task;

2) number of times the user did not continue;

3) number of actions taken that do not solve the problem;

4) number of minutes (hours) spent on one error recovery;

5) percentage of all time spent on error recovery; and

6) number of times the user has to reboot/start again.

Each time any one of these is not satisfied, we count one unit towards inadequate error recovery. The fuzzy-term set, therefore, is \{Good, Average, Poor\}. The fuzzy membership for error recovery is shown in Fig. 7.

\section{FUZZY INFERENCE SYSTEM FOR DETERMINATION OF USABILITY MEASURE}

In the last section, we developed a fuzzy model to express each of the usability features or factors that we believed were important in characterizing usability. What we still need is a method of mapping the input space corresponding to these features or factors to the output space that is a representation of usability. The input space here will have several dimensions, as explained earlier, and will consist of the features, system feedback, consistency, error prevention, performance/efficiency, user like/dislike, and error recovery. The output space will have a single dimension, namely the usability. The input features are 


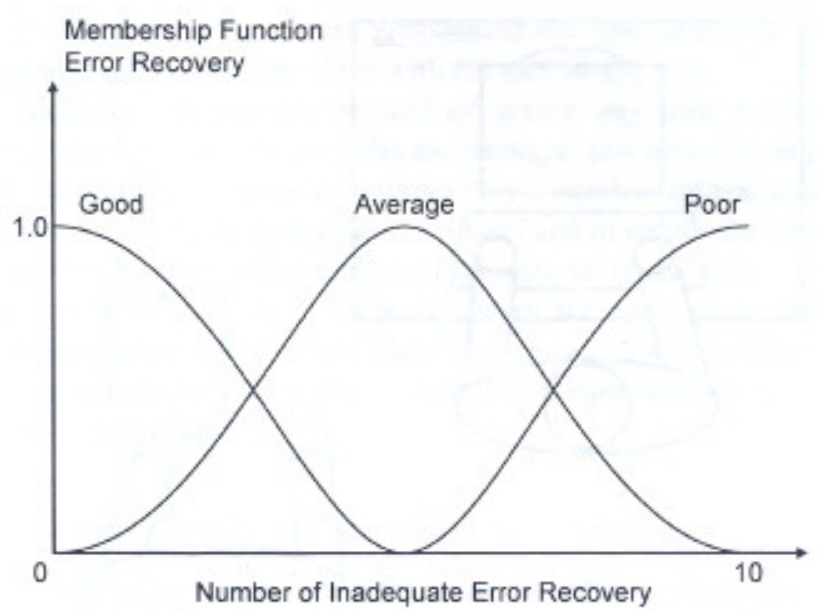

Fig. 7. Membership function for error recovery.

all expressed using a fuzzy model that transforms the variables of a particular input feature or factor into a linguistic variable. What we need is a method of fuzzy inference to perform the mapping from the input space to the output space. We will use fuzzy linguistic-control rules for performing this mapping. The general form of a fuzzy linguistic-control rule is as follows:

\section{If $A$ then $B$.}

This fuzzy linguistic-control rule states that if condition $A$ is met, then carry out action $B$. Sometimes, $A$ is referred to as the antecedent, and $B$ as the consequent. $A$ could be a composite condition or antecedent consisting of premises joined together by connectives AND and OR.

There are two broad categories of fuzzy-reasoning systems that need to be considered for our particular problem, and these are:

1) the Mamdani method [9];

2) the Takagi and Sugeno method [16].

In both approaches, the input conditions are given in the form of fuzzy sets partitioned on the input UoD. Further, in the Mamdani approach, the output is in the form of a fuzzy-set partition on the output UoD. In the case of the Takagi-Sugeno inference model, the output is a linear crisp function. Singletons represent a particular case of the Takagi-Sugeno model. This use of different forms for the output variable has considerable implications for the reasoning approach. In determining usability employing the fuzzy-systems approach, we have to choose between the two inference systems discussed above. What we would like to achieve is a value for the usability for this inference system that will allow us to have some measure of this property. Each of the above input factors influences the value of the output usability generated. We decided to choose the Takagi-Sugeno approach as the single crisp value returned, as a usability is a more practical approach in that it would be more helpful and understandable by a usability specialist. Further, there are several different approaches to aggregation and defuzzification that could result in somewhat different values for the output in the Mamdani approach, and this is likely to be confusing for the usability specialists. We can obtain from the test procedure a number of examples that provide values of inputs and a target value of usability. Methods of directly tuning the parameters in the Takagi-Sugeno model have been widely researched and they provide a reliable approach.

In the usability determination problem, it is, therefore, advantageous to use the Takagi-Sugeno inference system to allow this tuning of the parameters using the example data generated from the testing procedure in Section IV.

\section{A. Takagi-Sugeno Inference Approach}

The Takagi-Sugeno (T-S) form of the rule is

$$
\begin{aligned}
& \text { IF }\left(x_{1} \text { is } X_{1} \text { AND } x_{2} \text { is } X_{2}, \ldots, x_{n} \text { is } X_{n}\right) \\
& \operatorname{THEN}\left(y_{q}=a_{q 0}+a_{q 1} x_{1}+\ldots a_{q n} x_{n}\right)
\end{aligned}
$$$$
\text { WHERE: } x_{1}, x_{2} \text { are scalar inputs; } X_{1}, X_{2} \text { are fuzzy sets; }
$$$$
a_{q 0}, a_{q 1}, \ldots, a_{q n} \text {, are real numbers; and } y_{q} \text { is the consequent }
$$
of the rule.

This is the form of the fuzzy-logic rule that was initially proposed by Takagi-Sugeno in their original work [16]. In the singleton case, the output $y_{q}$ takes the form $y_{q}=a_{q 0}$.

We consider a system with $m$ fuzzy rules of the T-S form. The form of the crisp output is

$$
y(\underline{x})=\frac{\sum_{q=1}^{m} \alpha_{q}\left(a_{q 0}+\sum_{s=1}^{n} a_{q s} x_{s}\right)}{\sum_{q=1}^{m} \alpha_{q}} .
$$

Here, $\alpha_{q}$ is the firing strength of rule $q$. The actual approach to fuzzy reasoning, in this case, has the following steps:

1) fuzzify inputs;

2) obtain the firing strength $\alpha_{q}$ associated with each rule $q$;

3) obtain the output function of $y_{q}$ associated with each rule $q$ using the firing strength $\alpha_{q}$;

4) obtain the overall output $y(\underline{x})$ using the expressions in (1) given above.

\section{B. Fuzzy Rule Base for the Usability Problem}

As explained above, we intend to use the first-order Takagi-Sugeno approach for fuzzy inference with a linear function for the right-hand side. The inputs on the left-hand side of the fuzzy rule will consist of the factors or features that affect usability, as defined in Section II.

These factors or features are system feedback, consistency, error prevention, performance, error recovery, and user opinion. The input vector $\underline{x}$ is, therefore, defined to be $\underline{x}=[$ System Feedback, Consistency, Error Prevention, Performance, Error Recovery, User Opinion]. We will write this using short form notation as

$$
\underline{x}=\left[\begin{array}{lll}
\text { FB CO EP P ER UO] }
\end{array}\right.
$$


A typical premise would be of the form "Feedback is good." The structure of the left-hand side of the rule, therefore, is

IF (Feedback is $X_{1}$ ) AND

(Consistency is $X_{2}$ ) AND

(Error Prevention is $X_{3}$ ) AND

(Performance is $X_{4}$ ) AND

(Error Recovery is $X_{5}$ ) AND

(User Opinion is $X_{6}$ )

$X_{i} i=1, \ldots, 6$ denotes, in each case, the fuzzy sets

corresponding to the linguistic terms [Poor, Average, Good].

The actual form of the fuzzy sets, and the associated membership function in each case, were defined in Section II.

In short-form notation, the left-hand side of each of the rules would take the form

$$
\begin{aligned}
& \text { IF (FB is } \left.\left.X_{1}\right) \text { AND (CO is } X_{2}\right) \text { AND } \\
& \text { (EP is } X_{3} \text { ) AND (P is } X_{4} \text { ) AND } \\
& \text { (ER is } X_{5} \text { ) AND (UO is } X_{6} \text { ). }
\end{aligned}
$$

Since we are using the Takagi-Sugeno inference system, the right-hand side for the rule $q$ has the form Usability $y_{q}=a_{q o}+$ $a_{q 1} x_{1}+\ldots+a_{q 6} x_{6}$, where $x_{1}, \ldots, x_{6}$ are the input variables, i.e.,

$$
\text { Usability } \begin{aligned}
y_{q}= & a_{q 0}+a_{q 1} *(\text { Feedback }) \\
& +a_{q 2} *(\text { Consistency }) \\
& +a_{q 3} *(\text { Error Prevention }) \\
& +a_{q 4} *(\text { Performance }) \\
& +a_{q 5} *(\text { Error Recovery }) \\
& +a_{q 6} *(\text { User Opinion })
\end{aligned}
$$

or in short form notation

$$
\text { Usability } \begin{aligned}
& y_{q}=a_{q 0}+a_{q 1} * \mathrm{FB}+a_{q 2} * \mathrm{CO}+a_{q 3} * \mathrm{EP} \\
&+a_{q 4} * \mathrm{P}+a_{q 5} * \mathrm{ER}+a_{q 6} * \mathrm{UO} .
\end{aligned}
$$

Here $a_{q 0}, a_{q 1}, \ldots, a_{q 6}$ are parameters. A typical example of the left-hand side in short-form notation is

IF (FB is Good) AND (CO is Good) AND (EP is Good) AND ( $\mathrm{P}$ is Average) AND (ER is Good) AND (UO is Average).

The form of the $q$ th rule in the rule base, in short-form notation, therefore, is

$$
\begin{aligned}
& \text { IF (FB is } \left.X_{1}\right) \text { AND }\left(\mathrm{CO} \text { is } X_{2}\right) \text { AND }\left(\mathrm{EP} \text { is } X_{3}\right) \text { AND (P is } \\
& \left.\left.X_{4}\right) \text { AND (ER is } X_{5}\right) \text { AND }\left(\mathrm{UO} \text { is } X_{6}\right) \\
& \text { THEN } a_{q 0}+a_{q 1} * \mathrm{FB}+a_{q 2} * \mathrm{CO}+a_{q 3} * \mathrm{EP}+a_{q 4} * \\
& \mathrm{P}+a_{q 5} * \mathrm{ER}+a_{q 6} * \mathrm{UO} .
\end{aligned}
$$

The total number of possible fuzzy rules, if we have $n$ inputs and use $K$ fuzzy sets to span the UoD for each input, is equal to $K^{n}$. For the usability problem, therefore, if we use three fuzzy sets to span each input factor UoD, the total number

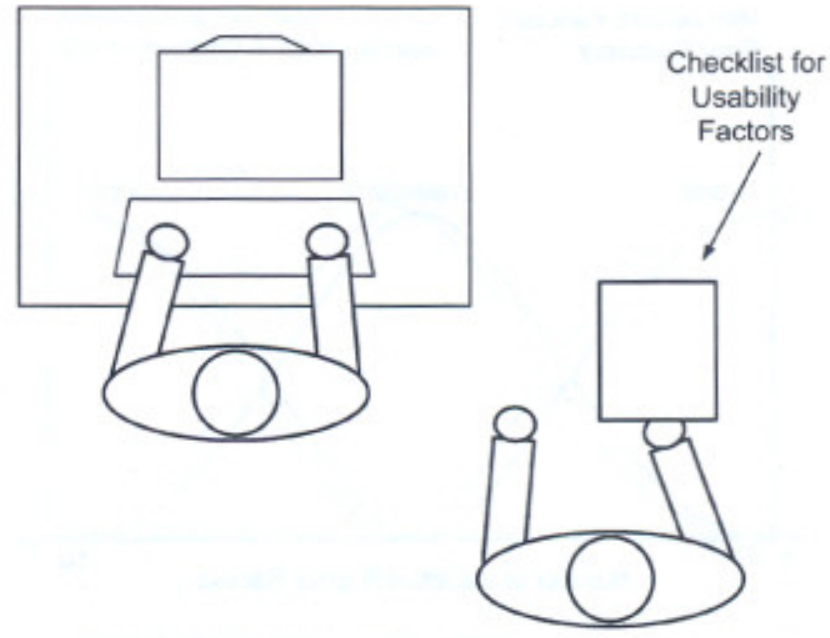

Fig. 8. Layout of scaled-down usability test.

of rules that we can have is $3^{6}=729$. This takes into consideration all possible combinations of the inputs. This is a rather large number of rules and many of these rules are redundant and, hence, will be deleted, in order to make the system more manageable. The building representation for AI using neural networks (BRAINNE) [14] will be used to prune the rules. Note that if we employed two fuzzy sets as shown in Fig. 2, then the total number of rules is $2^{6}=64$. Hence, as mentioned before, it is important to ascertain whether there is a significant gain in going to the three-fuzzy-set case, to justify the considerably increased computational effort.

\section{ObTaining TRaining Data}

The way in which we obtained the training data is to carry out usability testing. Essentially, this consists of letting a user test a particular piece of software by performing specific tasks on that software within a usability laboratory setup.

In order to understand this, we need to elaborate on

1) usability test layout and procedure;

2) software selection and task design;

3) test population.

\section{A. Usability Test Layout and Procedure}

The test layout consists of the test subject, the computer with the software being tested, and a test monitor as shown in Fig. 8 .

The test subject actually performs the given task on the software. The test monitor does not help or advise the test subject how to carry out any specific action. Rather, the test monitor observes what the test subject is doing and enters a score on a checklist that is used to determine units against each of the relevant input factors discussed previously. For example, each time the test subject makes an error, one unit is recorded against the number of items in the checklist. Note that the above is a fairly minimal test setup without any expensive video cameras, audio equipment, etc.

The test monitor performs the role of recording directly, rather than going through an intermediate phase of video or audio recording, followed by analysis of these. 
The test monitor always sits behind the user and is to be unobtrusive and not interfere with the user in any way.

The test procedure consisted of giving any user a short tutorial on how to use the software package, just going through the new functions. This gives them only a minimal introduction to the software, so as to orient the users and to reduce the time involved in otherwise reading tutorial material or manuals. This short tutorial was particularly necessary for some of the test subjects who used Windows-based interface software, as many of them were less computer literate than people who tested the UNIX-based UIs.

\section{B. Software Selection for the Tests and Task Design}

It was important in the selection of the software to be tested that we considered a range of interfaces, in order to have sufficiently representative data, which was used to tune the fuzzymetric parameters. To achieve this, we chose two classes of interfaces with several software packages being considered in each class. The first of these were for Microsoft-Windows-type environments and the second for UNIX-based environments.

In order to ensure that a good spread of software packages was used, software packages were chosen that are known to have good interfaces and are widely used in industry or on personal computers. These formed the "Good" category. We have also tested interfaces that came out of student softwareengineering projects, which have been developed by students in teams, getting experts to rate these as Average or Poor. Some of these were known to have particular defects with the interfaces, since they were designed without any reference to usability guidelines.

Lastly, there were several locally developed packages for commercial use that were also tested. Again, experts were asked to categorize these as Very Good, Good, Average, Poor, or Very Poor.

In addition to selecting particular software packages to test, we had to design specific tasks that were carried out by the test subject during the usability test for each of the different software packages. We designed these tasks based on the following principles; namely the tasks must be such that:

1) they must be work related so as to simulate the manner in which they would actually be used in the work environment;

2) they cover most of the significant functions of the package, so that all significant and frequently used features of the interface are tested;

3) the time of the test session clearly reflects work periods and is not too short as to be artificial or too long as to cause tiredness and fatigue to set in.

\section{Population of Users Tested}

The population of test subjects considered should be sufficiently representative of the final user population in the case of each software package. This could pose logistical difficulties in obtaining enough of each type of subject. A compromise was reached where we used several computer science students from each of the different year levels, some secretarial staff, and

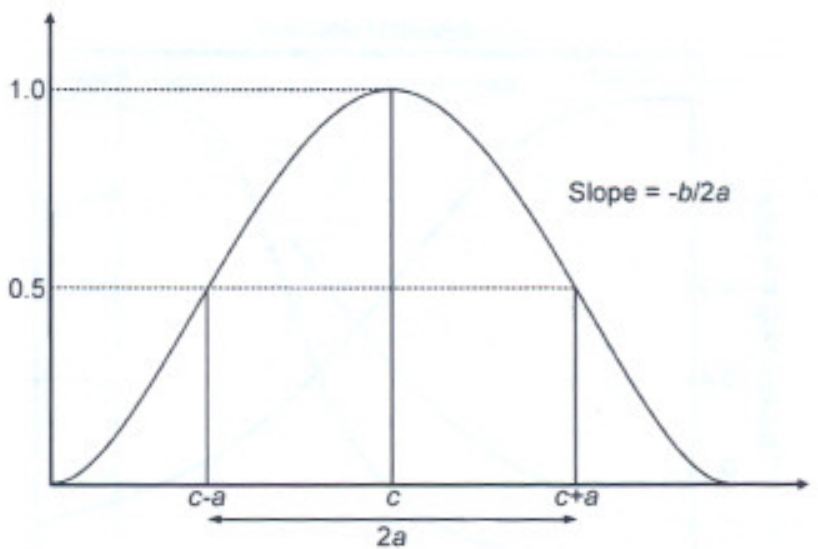

Fig. 9. Membership function showing parameters.

a small group of noncomputer-oriented users. The secretarial staff were included because the target population for some of the packages included this group. The students were chosen because they would find jobs in categories that would require the use of some of the UNIX-based packages. The noncomputeroriented users were included to give us a sufficient spread of users. In addition to the test subjects who were essentially unfamiliar with the software package they were testing, we also included one expert user on each package, so as to obtain some of the data necessary for calculating some of the input features.

\section{RESULTS OBTAINED AND DISCUSSION}

A set of data points were generated from testing several different UIs; the total number of actual tests carried out was 49. We generated several points around each of these test results by doing small perturbations of the input vectors to give a total of 622 independent points. These were then duplicated three times to give 1866 data points that could be used in conjunction with the tuning algorithms. The actual tuning algorithm used was the adaptive-network-based fuzzy-inference system (ANFIS) algorithm [20], since it tunes the parameters of both the input membership function, as well as the output coefficients. For the studies carried out in this paper, the version of ANFIS implemented in the Fuzzy Logic Toolbox of MATLAB was used. Since this is an experimental investigation of the fuzzy-logic-based usability metric, for the reasons outlined in Section III, it was decided to investigate the use of:

1) two fuzzy sets corresponding to the linguistic term set [Good, Bad] to span the input space for each of the six input factors (see Fig. 2 for feedback); and

2) three fuzzy sets corresponding to the linguistic term set [Good, Average, Bad] to span the input space of each of the six input factors.

Lastly, we will give a comparison of 1) and 2) above.

As explained earlier, the actual shape of the membership function used in these studies for each input factor is the G-Bell shape (i.e., the Gbellmf). The meaning of the parameters $[a, b, c]$, which characterize the G-Bell function are shown in Fig. 9 . 


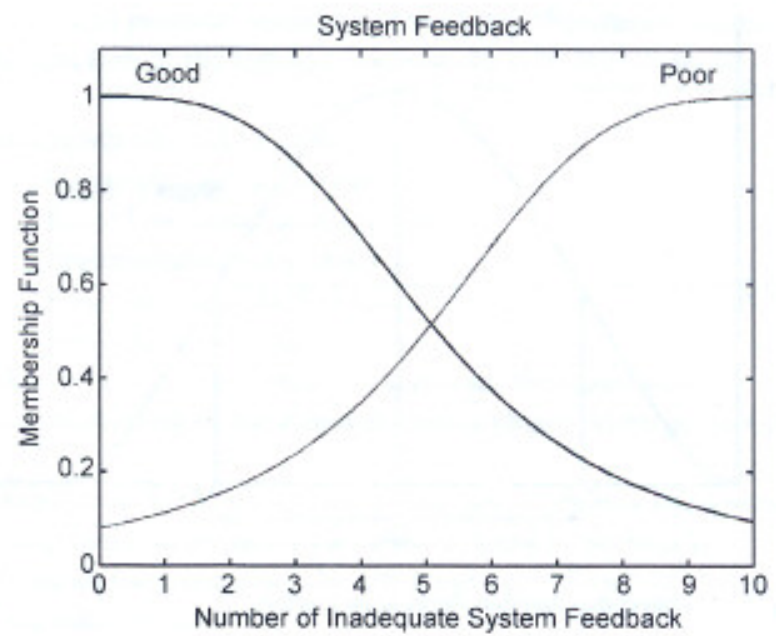

Fig. 10. Membership function for system feedback.

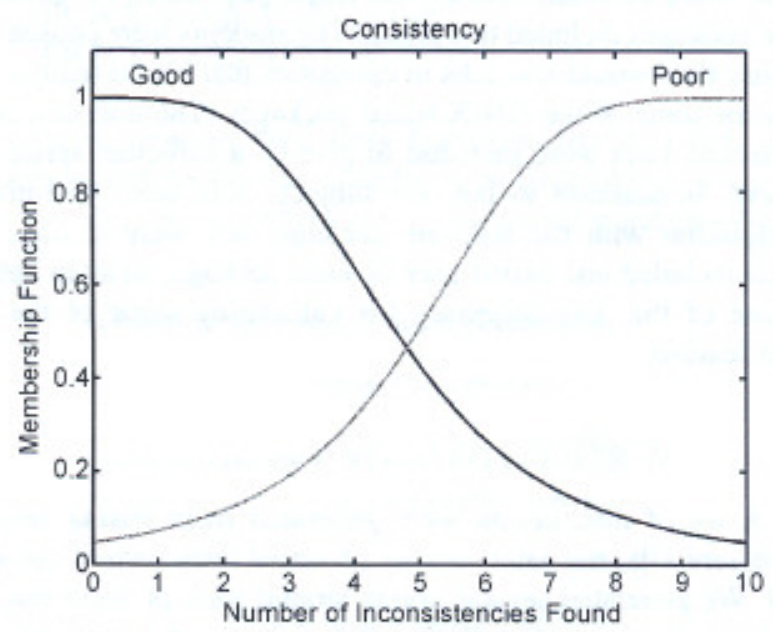

Fig. 11. Membership function for consistency.

The membership function is given by the expression

$$
\mu(x)=\frac{1}{\left[1+\left\{\left(\frac{x-c}{a}\right)^{2}\right\}^{b}\right]} .
$$

\section{A. Fuzzy Membership Functions for the Inputs}

The Fuzzy System ToolBox generates an initial set of values for parameters $[a, b, c]$, and then tunes them using the ANFIS model. Their values are given in the Appendix in Table IX for the two-fuzzy-set case and Table $\mathrm{X}$ for the three-fuzzy-set cases.

1) Two Fuzzy Sets Per Input Factor: The membership functions for the case of two fuzzy sets per input factor obtained are shown in Figs. 10-15

The "Poor" membership function of the input factor Error Prevention is moved to the right, and this is consistent with the training data. Many of the counts in the tests were generally fairly high, even for good interfaces. By shifting the membership functions to the right, the tuned FIS is trying to reduce

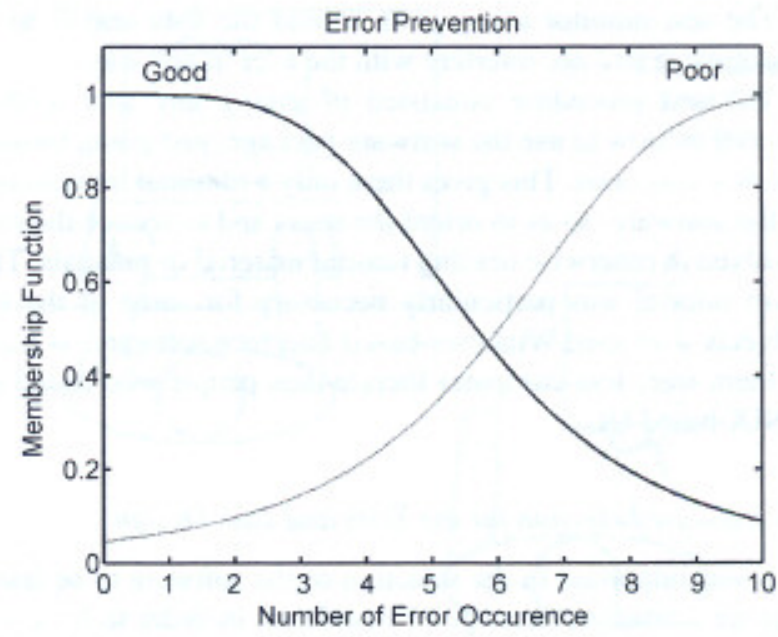

Fig. 12. Membership function for error prevention.

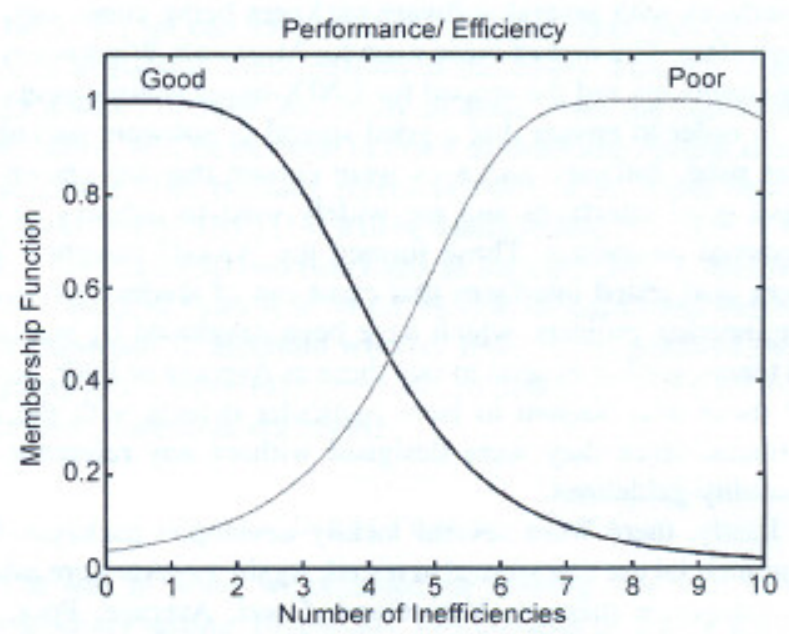

Fig. 13. Membership function for efficiency.

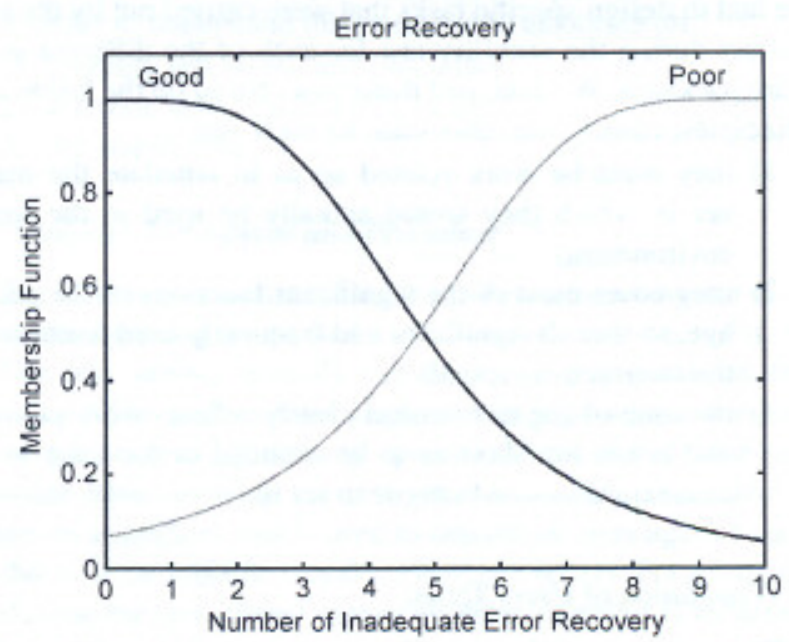

Fig. 14. Membership function for error recovery.

the range of the "Poor" subset, since even with moderately high counts of incidents of errors, an interface can still be considered to be "Good." In contrast, the Poor membership function is 


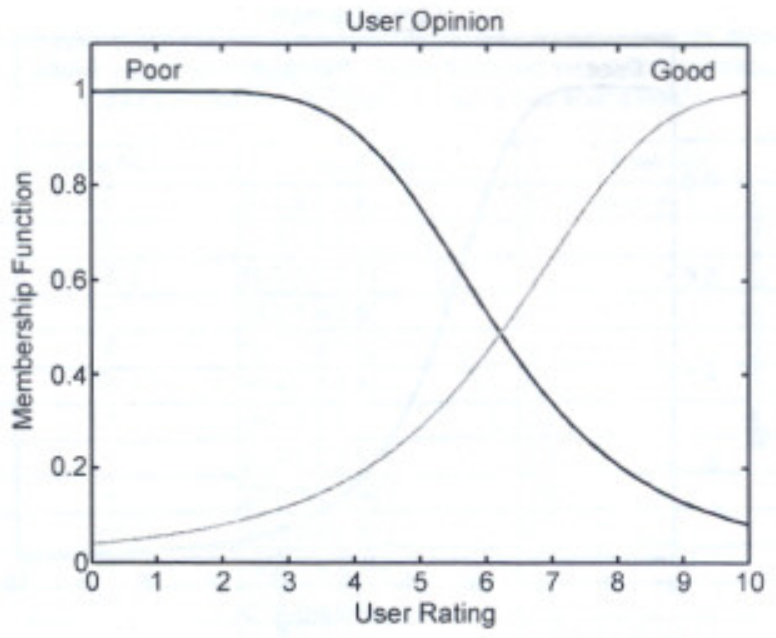

Fig. 15. Membership function for user likeability.

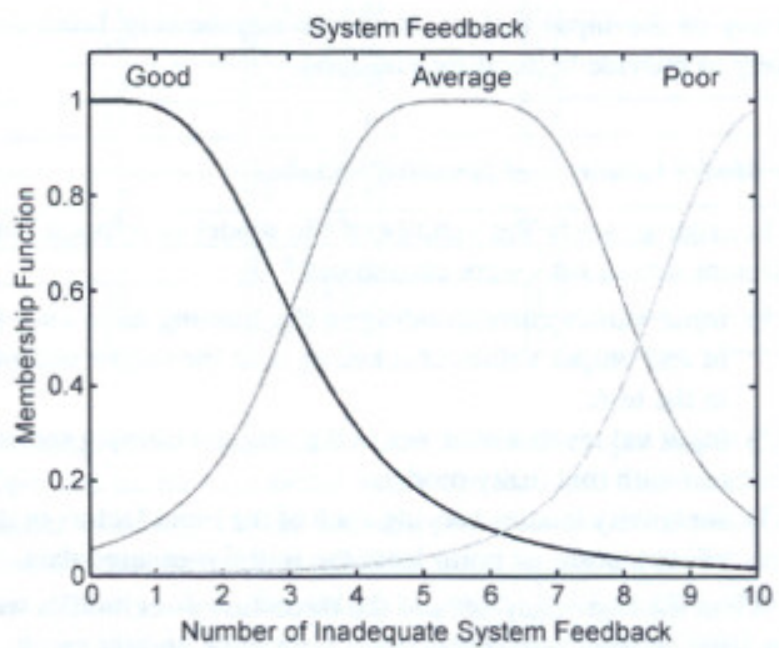

Fig. 16. Membership function for system feedback.

shifted slightly to the left for the input factor Consistency, indicating that the number of counts of inconsistencies for Good were not as high for the Poor interface. Good membership function is concentrated towards the lower counts of detected inconsistencies. This reduces the range for the Good subspace. This indicates that low scores for this factor were obtained for Good interfaces when compared with those obtained for Error Prevention. The reasons for this are that error prevention is essentially a complex process involving an understanding of the dynamics of the software, and the likely nonstandard actions that the user is likely to take. In contrast, consistency is more static and, therefore, it is easier for the user-interface designer to achieve a better score with this.

We also note that performance is skewed to the left. This means the poor interface has an expanded range and the good interface is compressed to having fewer inefficiencies. This implies that the user is able to perform very well on the good interface, but with a poor interface his/her performance can vary from reasonably well to very poor. This probably is a reflection of the competence of the user in dealing with the poor interface.

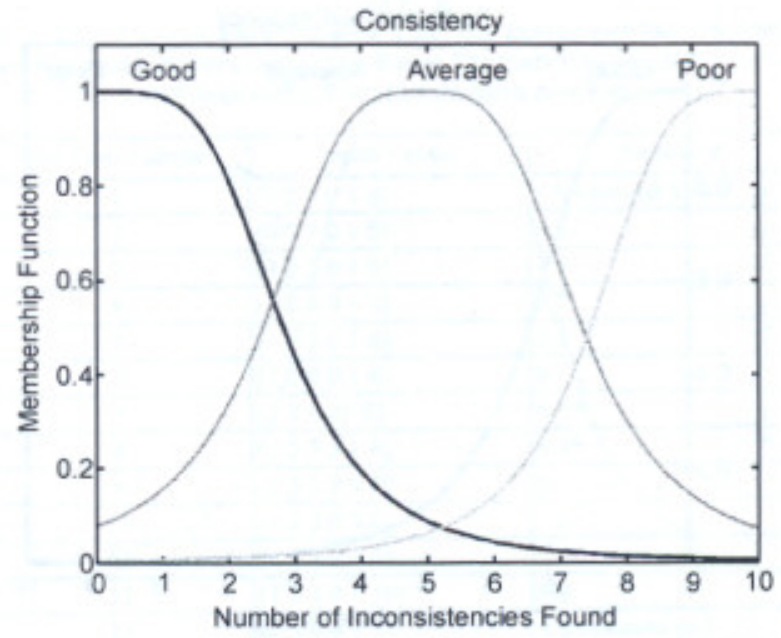

Fig. 17. Membership function for consistency.

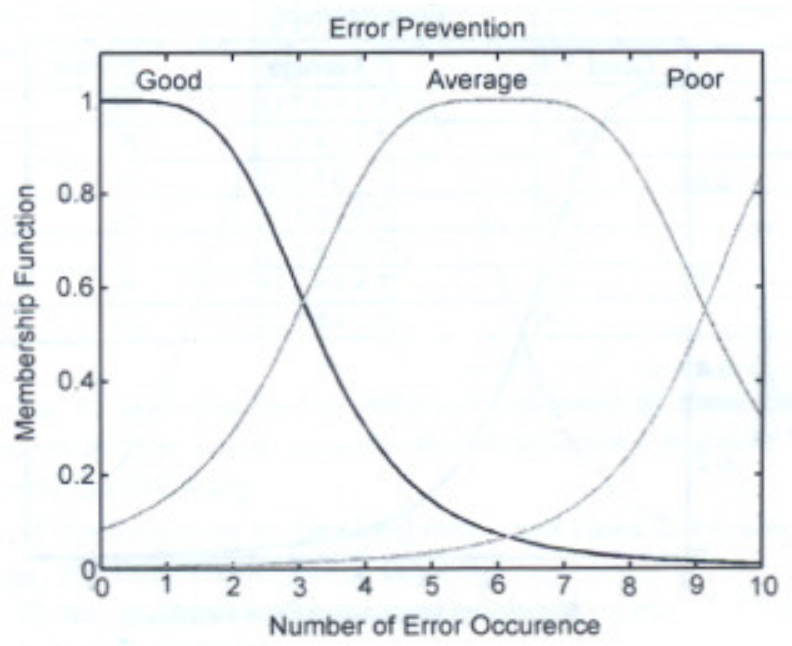

Fig. 18. Membership function for error prevention.

Lastly, we note that the membership functions for User Opinion have moved to the right. This indicates that users were willing to give the interfaces a higher score than that obtained from the experts.

There are $2^{6}=64$ rules in this T-S fuzzy model. Each of these rules will have seven output parameters associated with it, giving a total of $64 \times 7=448$ output parameters. These output parameters are also tuned by ANFIS. This completes the determination of the total fuzzy-inference model in this case.

2) Three Fuzzy Sets Per Input Factor Model: Table X gives the parameters $[a, b, c]$ for each of the three fuzzy sets corresponding to the linguistic terms Good, Average, Poor, which span the input space for each of the factors in turn. The membership functions for the case of these fuzzy sets per input factor obtained are shown in Figs. 16-21.

We note that Poor and Average membership functions for the input factors Feedback and Error Prevention are skewed to the right. Note that this is consistent with the training data. As explained before, this has the effect of reducing the range of the Poor subset, since on these two input factors, even the counts 


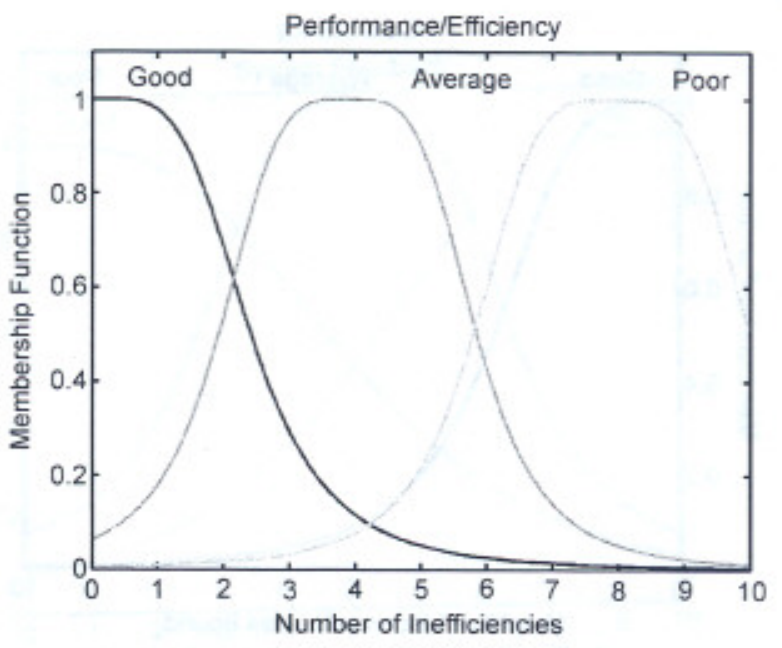

Fig. 19. Membership function for efficiency.

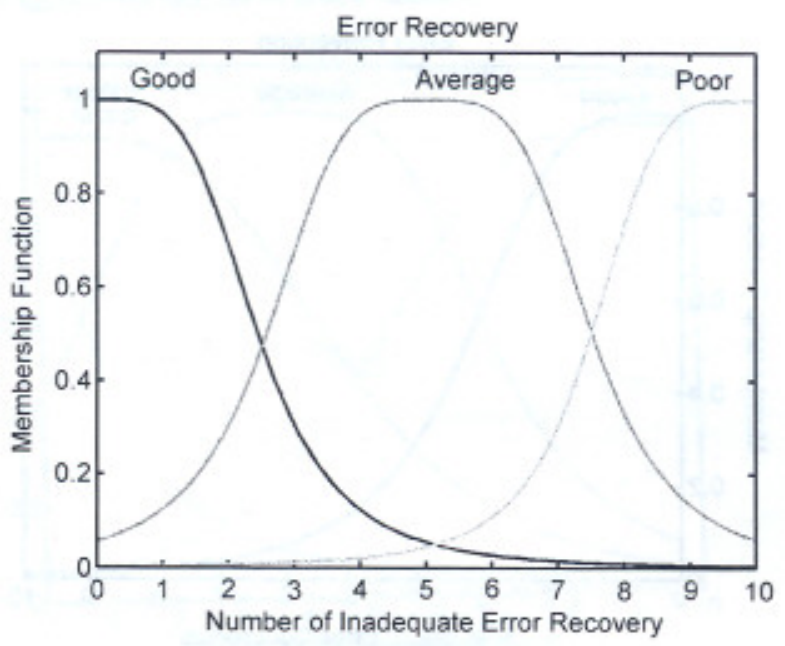

Fig. 20. Membership function for error recovery.

for the Good interfaces were moderately high. The membership functions for the input factors Consistency and Efficiency have been skewed to the left. Again, these results are consistent with those obtained for the two-membership-function case. Lastly we note the membership functions for Good, Average, and Poor for the input factor user opinion are moved to the right consistent with the results obtained for the two-membership-function case. The reasons for these effects are similar to those given for the two-membership-function case.

In all, the total number of rules in the three-membershipfunction case should be $3^{6}=729$ rules. However, as explained earlier, the number of rules are pruned using the BRAINNE approach. The final number of rules used with the model for this study was 38 and they are given in [3] and [21]. This gives a total of $38 \times 7=266$ output parameters. This completes the determination of the fuzzy-system model for this case.

3) Comparison of the Two-Fuzzy-Set and Three-Fuzzy-Set Cases: When we examine the two-fuzzy-set and three-fuzzyset membership functions, there is a much clearer separation between the good and poor membership functions for the threefuzzy-set case. So, if one wanted to use a measure with respect

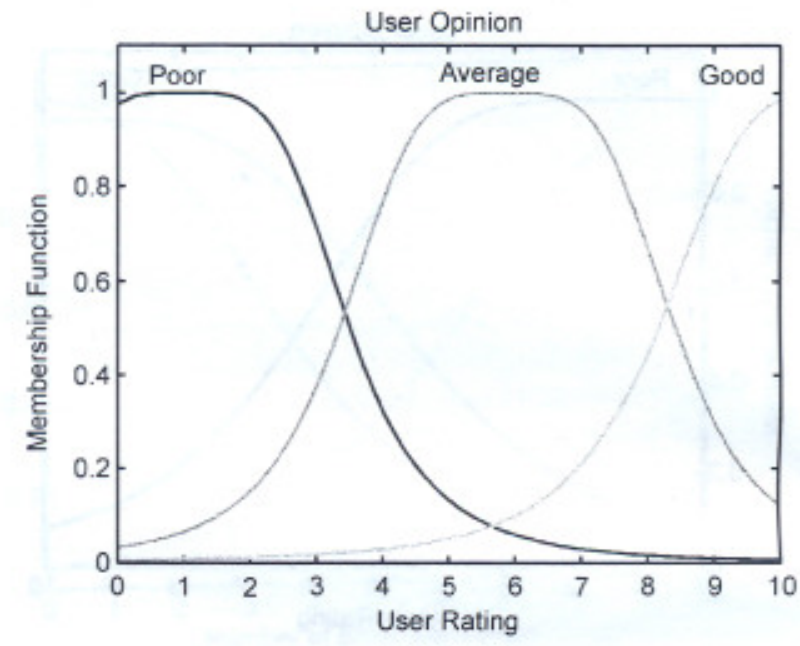

Fig. 21. Membership function for user likeability.

to any of the input factors, the three-membership function is likely to provide better differentiation.

\section{B. Model Validity and Sensitivity Studies}

In order to study the validity of the model developed, three different sets of runs were carried out:

1) input values corresponding to the training data were fed in and output values checked against the output obtained in the test;

2) input values that were not in the original training set were run with this fuzzy model;

3) sensitivity studies varying each of the input factors in turn about a nominal point from the initial measured data.

When the two-fuzzy-set and the three-fuzzy-set models were run with original test values, they both gave correct results to within an acceptable tolerance for the overall figure of merit for usability. Similarly, when these two models were run with additional test points, they also both gave correct results to within an acceptable tolerance for the overall figure of merit for usability.

1) Sensitivity Studies for Two-Fuzzy-Set Model: The results for the sensitivity studies were obtained by choosing two different nominal points for each of the good, average, and poor interfaces, and then perturbing the input factors about each of these nominal points. The two-fuzzy-set model is then used to find the overall measure of usability for each of these perturbed values. Thus, in Table I for the good interface, the nominal point has input vector [ $\left[\begin{array}{llllll}0 & 4 & 5 & 4 & 3 & 8\end{array}\right]$ corresponding to a usability of 7.5. The first perturbation increases the system feedback to 2 . This corresponds to a deterioration in system feedback leading to a drop in overall usability to 7.3 .

The results for the sensitivity studies are given in Tables I-IV; the input factors refer to the scores obtained for the following components of the vector: [Feedback, Consistency, Error Prevention, Performance, Error Recovery, User Opinion].

Note that the score indicates the number of deficiencies in the first five input factors and, hence, a higher score indicates a poorer score for that particular input factor and, hence, a lower 
TABLE I

INPUT TEST CASES DERIVED FROM VARYING DIFERENT INPUTS ABOUT TWo Nominal Points OBtained From Testing THE GOOD INTERFACE (FIRST CATEGORY). TWO FUZZY SETS FOR EACH INPUT

\begin{tabular}{|c|c|c|}
\hline Test Case Number & Input Vector & \begin{tabular}{|r} 
Usability \\
\end{tabular} \\
\hline 1 & $\mid 0454381$ & 7.5 nominal pt I \\
\hline 2 & $|245438|$ & 7.3 \\
\hline 3 & {$\left[\begin{array}{llllllllllll}0 & 6 & 5 & 4 & 3 & 8\end{array}\right]$} & 7 \\
\hline 4 & 10254381 & 7.8 \\
\hline 5 & {$[047438]$} & 5,8 \\
\hline 6 & {$\left[\begin{array}{llllll}0 & 4 & 3 & 4 & 3 & 8\end{array}\right]$} & 8.2 \\
\hline 7 & {$\left[\begin{array}{llllll}0 & 4 & 5 & 6 & 3 & 8\end{array}\right]$} & 7.1 \\
\hline 8 & {$\left[\begin{array}{llllll}0 & 4 & 5 & 2 & 3 & 8\end{array}\right]$} & 7.9 \\
\hline 9 & {$[045458$ ] } & 7.3 \\
\hline 10 & {$\left[\begin{array}{llllll}0 & 4 & 5 & 4 & 1 & 8\end{array}\right]$} & 8.1 \\
\hline 11 & {$\left[\begin{array}{lllll}04 & 5 & 4 & 3 & 10\end{array}\right.$} & 7.9 \\
\hline 12 & $\mid 045436]$ & 7.2 \\
\hline 13 & {$[1555526]$} & 7.6 nominal pt 2 \\
\hline 14 & $|055526|$ & 7,7 \\
\hline 15 & {$[3555526]$} & 6.8 \\
\hline 16 & {$[135526]$} & 7.6 \\
\hline 17 & {$[165526]$} & 7.1 \\
\hline 18 & {$[153526]$} & 7.6 \\
\hline 19 & {$[157526]$} & 6.6 \\
\hline 20 & {$\left[\begin{array}{llllll}1 & 5 & 5 & 3 & 2 & 6\end{array}\right]$} & 7.0 \\
\hline 21 & {$\left[\begin{array}{llll}5 & 5 & 5 & 726 \\
\end{array}\right.$} & 7 \\
\hline 22 & {$[155506]$} & 7.3 \\
\hline 23 & {$[155556]$} & 5.3 \\
\hline 24 & 11555231 & 7.2 \\
\hline 25 & {$\left[\begin{array}{lllllllll}1 & 5 & 5 & 5 & 2 & 8\end{array}\right]$} & 7.6 \\
\hline
\end{tabular}

usability. However, a higher score in the last input factor (User Opinion) indicates a better score for this factor and, hence, a higher usability. Let us initially consider all the results, and then the results with respect to some of the input factors in turn. Nearly all of the values of usability generated indicate a movement in the correct direction for a perturbation in a single input factor about the nominal point. The exceptions are indicated by an asterisk $(*)$. However, even for these, while the movement with respect to the nominal point might be slightly in the wrong direction, if two adjacent points corresponding to a change in the same input factor are considered, we notice three of these points indicate a movement in the correct direction namely Test 20 and Test 22 (Table I), and Test 20 (Table II).

Let us consider the value of usability for a Good interface is approximately between 7 and 10 , Average interface approximately between 3.0 and 6.9 , and a Poor interface approximately 2.9 or below. Note that in reality, each of these will be fuzzy sets. However, for the purposes of the qualitative discussion below the above ranges will suffice. Thus, we note that even the remaining exception points do not lead to a change in category of output that is wrong. These exception points could result from abnormal responses from a few users and because there are not many results from users, they are insufficient to filter these out.

All the remaining points obtained, which are the large majority, lead to a change in usability in the expected direction. Hence, in broad terms, these two-membership-function fuzzy models seem to adequately model the relationship between these input factors and the overall usability.
TABLE II

INPUT TEST CASES DERIVED FroM VARYING DIFFERENT INPUTS ABOUT Two Nominal Points Obtained From Testing the Good Interface (SECOND CATEGORY). TWO FUZZY SETS FOR EACH INPUT

\begin{tabular}{|c|c|c|}
\hline Test Case Number & Input Vector & Usability \\
\hline 1 & {$\left[\begin{array}{lllllll}1 & 2 & 3 & 0 & 1 & 8\end{array}\right]$} & 7.9 nominal pt 1 \\
\hline 2 & {$\left[\begin{array}{lllllll}0 & 2 & 3 & 0 & 1 & 8\end{array}\right]$} & 8.2 \\
\hline 3 & {$\left[\begin{array}{lllllll}3 & 2 & 3 & 0 & 1 & 8\end{array}\right]$} & 7.7 \\
\hline 4 & {$\left[\begin{array}{lllllll}1 & 0 & 3 & 0 & 1 & 8\end{array}\right]$} & 8.7 \\
\hline 5 & {$\left[\begin{array}{lllllll}1 & 5 & 3 & 0 & 1 & 8\end{array}\right]$} & 6.1 \\
\hline 6 & {$\left[\begin{array}{llllll}1 & 2 & 0 & 0 & 1 & 8\end{array}\right]$} & 9 \\
\hline 7 & {$\left[\begin{array}{lllllll}1 & 2 & 6 & 0 & 01 & 8\end{array}\right.$} & $9.5^{*}$ \\
\hline 8 & {$\left[\begin{array}{llllll}1 & 2 & 3 & 3 & 1 & 8\end{array}\right]$} & $8.3^{*}$ \\
\hline 9 & {$\left[\begin{array}{llllll}1 & 2 & 3 & 0 & 0 & 8\end{array}\right]$} & 8.2 \\
\hline 10 & {$\left[\begin{array}{lllll}12 & 23 & 0 & 3 & 8\end{array}\right.$} & 7.6 \\
\hline 11 & {$[12230016]$} & 7.4 \\
\hline 12 & {$\left[\begin{array}{lllllll}1 & 2 & 3 & 0 & 0 & 1 & 10\end{array}\right.$} & 8.8 \\
\hline 13 & {$[234417]$} & 8.0 nominal pt 2 \\
\hline 14 & {$[034417 \mid$} & 8.2 \\
\hline 15 & {$\left[\begin{array}{lllllll}4 & 3 & 4 & 4 & 1 & 7\end{array}\right]$} & 7.1 \\
\hline 16 & 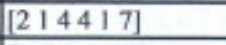 & 8.4 \\
\hline 17 & {$\left[\begin{array}{lllllll}2 & 5 & 4 & 4 & 1 & 7\end{array}\right]$} & 7.3 \\
\hline 18 & {$\left[\begin{array}{lllllll}2 & 3 & 1 & 4 & 1 & 7\end{array}\right]$} & 8.7 \\
\hline 19 & {$\left[\begin{array}{llllll}2 & 3 & 6 & 4 & 1 & 7\end{array}\right.$} & 7.1 \\
\hline 20 & {$\left[\begin{array}{llllllll}2 & 3 & 4 & 1 & 1 & 7 & 7\end{array}\right.$} & $7.8^{\circ}$ \\
\hline 21 & {$\left[\begin{array}{lllllll}2 & 3 & 4 & 6 & 1 & 1 & 7\end{array}\right]$} & 7.3 \\
\hline 22 & {$[2344407]$} & 8.3 \\
\hline 23 & {$\left[\begin{array}{lllllll}2 & 3 & 4 & 4 & 3 & 7\end{array}\right]$} & 7.6 \\
\hline 24 & {$[234447]$} & 7.4 \\
\hline 25 & {$[2344415]$} & 6.9 \\
\hline
\end{tabular}

Let us next discuss the influences of some of these input factors in turn. Let us consider the input factor Feedback. We notice the following:

1) for interfaces in the Good range, test cases 2, 14, and 15 (in Table I), and 14 (in Table II).

2) for interfaces in the Average range, test cases 2, 3, 15, and 16 (in Table III).

3) for interfaces in the Poor range, test case 3 (Table IV).

All indicate that an improvement in Feedback (reduced score) leads to an improvement (higher value) in Usability, and a deterioration in Feedback (increased score) leads to a deterioration (lower score) in Usability. Further, we note in test case 15 (Table I) that the categorization of interface falls from the Good towards the Average category range for a two-point increase in score in Feedback deficiencies. This indicates that Feedback has an important influence on the usability. However, in some cases, the influence is more pronounced (e.g., test case 15-Table III).

Let us consider the input factor Consistency and note the following:

1) for interfaces in the Good usability range, test cases 3, 4, and 15 (Table I), test cases 4, 5, 16, and 17 (Table II);

2) for interfaces in the Average usability range, test cases 4 , 5,17 , and 18 (Table III);

3) for interfaces in the Poor usability range, test cases 4,5 , 17, and 18 (Table IV).

All indicate that an improvement in Consistency leads to an improvement in usability, and vice versa. Further, we notice that for test case 5 (Table II), a change (deterioration) in the 
TABLE III

INPUT TEST CASES DERIVED FROM VARYING DIFFERENT INPUTS ABOUT TWO NOMINAL POINTS OBTAINEd FroM TESTING THE AVERAGE INTERFACE. TWO FUZZY SETS FOR EACH INPUT

\begin{tabular}{c|l|l}
\hline Tesi Case Number & \multicolumn{1}{|c|}{ Input Vector } & \multicolumn{1}{c}{ Usability } \\
\hline I & {$[557548]$} & 6.0 nominal pt I \\
\hline 2 & {$[357548]$} & 6.3 \\
\hline 3 & {$[757548]$} & 5.2 \\
\hline 4 & {$[537548]$} & 6.7 \\
\hline 5 & {$[587548]$} & 5.5 \\
\hline 6 & {$[559548]$} & 5.6 \\
\hline 7 & {$[554548]$} & 5.8 \\
\hline 8 & {$[557348]$} & $3.4^{*}$ \\
\hline 9 & {$[557748]$} & $6.8^{*}$ \\
\hline 10 & {$[557528]$} & 6.2 \\
\hline 11 & {$[557568]$} & 5.3 \\
\hline 12 & {$[5575410]$} & 6.3 \\
\hline 13 & {$[557546]$} & 5.9 \\
\hline 14 & {$[457637]$} & 5.9 nominal pt 2 \\
\hline 15 & {$[157637]$} & 7.6 \\
\hline 16 & {$[657637]$} & 5.5 \\
\hline 17 & {$[437637]$} & 6.6 \\
\hline 18 & {$[447637]$} & 5.9 \\
\hline 19 & {$[455637]$} & 5.9 \\
\hline 20 & {$[459637]$} & 5.6 \\
\hline 21 & {$[457337]$} & 6.1 \\
\hline 22 & {$[457837]$} & 5.7 \\
\hline 23 & {$[457617]$} & 6.4 \\
\hline 24 & {$[457607]$} & 6.8 \\
\hline 25 & {$[457667]$} & 3.6 \\
\hline & &
\end{tabular}

Consistency score from 2 to 5 takes the interface from the Good to the Average range. Further, for test case 4 (Table IV), a change in the Consistency score from 7 to 5 (improvement) takes the UI from the Poor to the Average usability range. In all other cases above, the change in the value for the Consistency score does not lead to a change in the usability category range. However, it does lead to a change in the usability value in the correct direction. This implies that Consistency does have a significant influence on usability. However, its influence is also tempered by the scores for the other input factors, and the initial level at which the consistency score was.

Let us next consider the input factor User Opinion, and we note the following:

1) for interfaces in the Good range: test cases 11, 12,24, and 25 (Table I), test cases 11, 12, 25, 26, and 27 (Table II);

2) for interfaces in the Average range: test cases 12, 13,26, and 27 (Table III);

3) for interfaces in the Poor range: test cases 12, 13, 25, and 26 (Table IV).

All indicate that an improvement in user opinion leads to an improvement in the usability value, and vice versa. In three of the cases, it also leads to a change in category range of the interface when the user opinion changes, and these are: test case 25 (Table II) from Good to Average; test case 27 (Table II) from Good to Poor; test case 13 (Table IV) from Poor to Average. User opinion of the interface appears to be a very significant factor in the determination of overall figure of merit of usability. However, the extent of its influence appears to be moderated by the other input factors.
TABLE IV

INPUT TEST CASES DERIVED From VARYING DiffERENT INPUTS ABOUT TWO NOMINAL. POINTS OBTAINED From TESTING THE POOR INTERFACE. TWO FUZZY SETS FOR EACH INPUT

\begin{tabular}{|c|c|c|}
\hline Test Case Number & Input Vector & Usability \\
\hline 1 & {$[9711692]$} & 2.0 nominal pt 1 \\
\hline 2 & {$[6711692]$} & $1.6^{*}$ \\
\hline 3 & {$\left[\begin{array}{llllllll}10 & 7 & 11 & 6 & 9 & 2\end{array}\right]$} & 1.9 \\
\hline 4 & {$[9511692]$} & 4.4 \\
\hline 5 & {$[9911692]$} & 1.2 \\
\hline 6 & {$[978692]$} & 2 \\
\hline 7 & 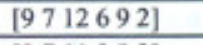 & 1.7 \\
\hline 8 & {$\left[\begin{array}{llllll}9 & 7 & 1 & 1 & 3 & 9\end{array}\right]$} & 6.1 \\
\hline 9 & {$\left[\begin{array}{llllll}9 & 7 & 11 & 8 & 9 & 2\end{array}\right]$} & 1.5 \\
\hline 10 & {$\left[\begin{array}{llllll}9 & 7 & 11 & 6 & 72\end{array}\right]$} & 1.7 \\
\hline 11 & {$\left[\begin{array}{lllllll}9 & 7 & 11 & 6 & 10 & 2\end{array}\right]$} & 2 \\
\hline 12 & {$[9711690]$} & 2 \\
\hline 13 & $\{9711695]$ & 3.3 \\
\hline 14 & {$[789672]$} & 2.0 nominal pt 2 \\
\hline 15 & 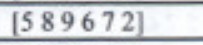 & 3 \\
\hline 16 & {$\left[\begin{array}{lllll}9 & 8 & 9 & 6 & 72\end{array}\right]$} & 1.7 \\
\hline 17 & {$\left[\begin{array}{l}769 \\
\end{array}\right.$} & 2,4 \\
\hline 18 & {$[7109672]$} & 1.8 \\
\hline 19 & {$[787672]$} & 3 \\
\hline 20 & {$[7811672]$} & 1.8 \\
\hline 21 & {$[789472]$} & 4 \\
\hline 22 & {$[789872]$} & 1.5 \\
\hline 23 & {$\left[\begin{array}{l}789 \\
\end{array}\right.$} & 5.8 \\
\hline 24 & {$[789692]$} & 1.8 \\
\hline 25 & {$[789671]$} & 1.8 \\
\hline 26 & {$[789675]$} & 2.9 \\
\hline
\end{tabular}

When we examine the other input factors, Error Prevention, Performance, and Error Recovery, we note that in each case, an improvement in any of these input factors leads to an improvement in usability, and vice versa. However, in one test case, for each input factor, a perturbation in the input factor leads to a change in usability. Hence, while these factors have an influence on usability, it does not appear to be as strong as user opinion or consistency.

2) Sensitivity Studies for Three-Fuzzy-Set Model: We present the results for the sensitivity studies on the three-fuzzyset model. These are obtained, as before, by varying the input factors around a measured nominal point. These results are presented in Tables V-VIII.

Most of the values of usability generated indicate a movement in the correct direction for a perturbation in a single input factor about the nominal point. The exceptions are indicated by an asterisk (*). Again for these, while the movement with respect to the nominal point might be slightly in the wrong direction, if two adjacent points corresponding to a change in the same input factor are considered, we notice that eight of these points indicate a movement in the correct direction namely: test cases $6,16,20$, and 22 (Table V), test cases 4 . 5 , and 20 (Table VI), test cases 9, 17, and 21 (Table VII), and test cases $3,4,10$, and 11 (Table VIII).

Furthermore, note that even the remaining exception points do not lead to a change in the category of usability range that is wrong. The reasons for these exception points have been discussed in the previous section. All the remaining points, 
TABLE V

INPUT TEST CASES DERIVED FROM VARYING DIFFERENT INPUTS ABOUT Two Nominal Points Output Training Pairs ObTained From TESTING THE GOOD INTERFACE (FIRST CATEGORY). THREF FUZZY SETS FOR EACH INPUT

\begin{tabular}{c|l|l}
\hline Test Case Number & \multicolumn{1}{|c|}{ Input Vector } & \multicolumn{1}{c}{ Usability } \\
\hline 1 & {$[045438]$} & 7.4 nominal pt I \\
\hline 2 & {$[245438]$} & 7.2 \\
\hline 3 & {$[065438]$} & 6.9 \\
\hline 4 & {$[025438]$} & 7.8 \\
\hline 5 & {$[047438]$} & 7.4 \\
\hline 6 & {$[043438]$} & $7.1^{*}$ \\
\hline 7 & {$[045638]$} & 7.3 \\
\hline 8 & {$[045238]$} & 10 \\
\hline 9 & {$[045458]$} & 7.4 \\
\hline 10 & {$[045418]$} & 8.1 \\
\hline 11 & {$[0454310]$} & 8 \\
\hline 12 & {$[045436]$} & 7.3 \\
\hline 13 & {$[155526]$} & 7.6 nominal pt 2 \\
\hline 14 & {$[055526]$} & 7.6 \\
\hline 15 & {$[355526]$} & 7.1 \\
\hline 16 & {$[135526]$} & $7.4^{*}$ \\
\hline 17 & {$[165526]$} & 7.3 \\
\hline 18 & {$[153526]$} & 8.7 \\
\hline 19 & {$[157526]$} & 7.3 \\
\hline 20 & {$[155326]$} & $7.1^{*}$ \\
\hline 21 & {$[155726]$} & 6.1 \\
\hline 22 & {$[155506]$} & $7.3^{*}$ \\
\hline 23 & {$[155556]$} & 6.3 \\
\hline & &
\end{tabular}

which constitute the large majority, lead to a change of usability in the expected direction. Hence, in general, this threemembership-function fuzzy model seems to adequately model the relationship between the input factors and usability.

3) Three Fuzzy Sets Per Input Factor Mode: Let us next consider the input factor User Opinion, and note that:

1) for interfaces in the Good range: test cases 11, 12, 24, and 25 (Table V), test cases 11, 12, 25, 26, and 27 (Table VI)

2) for interfaces in the Average range: test cases 12, 13, 26, and 27 (Table VII);

3) for interfaces in the Poor range: test cases 12, 13, 25, and 26 (Table VIII).

All indicate that an improvement in User Opinion leads to an improvement in the usability value, and vice versa. Further, we note that two points, test cases 11 and 27 (Table VI), change the usability category from the Good to the Average range. This corresponds to a change in User Opinion scores and membership values. For test case 11, User Opinion score ( 8 to 6 ), membership value approximately 0.5 (Good), 0.5 (Average) to 1.0 (Average). For test case 27, User Opinion score (5 to 2), membership value approximately 0.8 (Average) to 1.0 (Poor). These account for the changes in the usability for these two cases. User Opinion is a very significant indicator of usability, but the extent of its influence is moderated by the scores of the other input factors. This is similar to the results observed in the two membership function cases, however, here, three points changed category.

For the input factors Error Prevention, Performance, and Consistency, only one test case changes category when the factor is perturbed. For the input factors System Feedback and
TABLE VI

INPUT TEST CASES DERIVED FroM VARYING DIFFERENT INPUTS ABOUT TWo Nominal Points OUTPUT TRAining Pairs OBTAINEd From TESTING THE GOOD INTERFACE (SECOND CATEGORY). THREE FUZZY SETS FOR EACH INPUT

\begin{tabular}{|c|c|c|}
\hline Test Case Number & Input Vector & Usability \\
\hline 1 & {$\left[\begin{array}{lllll}1 & 2 & 3 & 0 & 1\end{array}\right.$} & 8.0 nominal pt 1 \\
\hline 2 & {$\left[\begin{array}{lllllll}0 & 2 & 3 & 0 & 1 & 8\end{array}\right]$} & 8.2 \\
\hline 3 & {$\left[\begin{array}{lllllll}3 & 2 & 3 & 0 & 1 & 8\end{array}\right]$} & 7.6 \\
\hline 4 & {$\left[\begin{array}{lllllll}1 & 0 & 3 & 0 & 1 & 8\end{array}\right]$} & $7.4^{*}$ \\
\hline 5 & 11530181 & $9.7^{*}$ \\
\hline 6 & {$\left[\begin{array}{lllllllllllll}1 & 2 & 0 & 0 & 0 & 1 & 8\end{array}\right]$} & 10 \\
\hline 7 & {$\left[\begin{array}{llllll}1 & 2 & 6 & 0 & 1 & 8\end{array}\right]$} & 4 \\
\hline 8 & {$\left[\begin{array}{lllllll}1 & 2 & 3 & 3 & 1 & 8\end{array}\right]$} & 8 \\
\hline 9 & {$\left[\begin{array}{llllll}1 & 2 & 3 & 0 & 0 & 0\end{array}\right]$} & 8.2 \\
\hline 10 & {$\left[\begin{array}{llllll}1 & 2 & 3 & 0 & 3 & 8\end{array}\right]$} & 5 \\
\hline 11 & 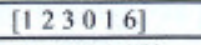 & 6.6 \\
\hline 12 & {$[1230110\rfloor$} & 9.4 \\
\hline 13 & {$[234417]$} & 7.9 nominal pt 2 \\
\hline 14 & {$\left[\begin{array}{lllllll}0 & 3 & 4 & 4 & 1 & 7\end{array}\right]$} & 8 \\
\hline 15 & {$[434417\rceil$} & 7.2 \\
\hline 16 & {$\left[\begin{array}{lllllll}2 & 1 & 4 & 4 & 4 & 7\end{array}\right]$} & 9 \\
\hline 17 & $|254417|$ & 7.5 \\
\hline 18 & {$\left[\begin{array}{lllllll}2 & 3 & 1 & 4 & 1 & 7\end{array}\right]$} & 9.9 \\
\hline 19 & {$\left[\begin{array}{llllll}2 & 3 & 6 & 4 & 1 & 7\end{array}\right]$} & 7.8 \\
\hline 20 & {$\left[\begin{array}{llllll}2 & 3 & 4 & 1 & 1 & 7\end{array}\right]$} & $7.7^{*}$ \\
\hline 21 & {$\left[\begin{array}{lllllll}2 & 3 & 4 & 6 & 1 & 7\end{array}\right]$} & 7.7 \\
\hline 22 & {$[234407]$} & 8 \\
\hline 23 & {$\left[\begin{array}{llllll}2 & 3 & 4 & 4 & 3 & 7\end{array}\right]$} & 7.6 \\
\hline 24 & {$[234447 \mid$} & 7.3 \\
\hline 25 & {$\left[\begin{array}{llllll}2 & 34 & 4 & 1 & 5\end{array}\right]$} & 7.6 \\
\hline
\end{tabular}

Error Recovery, none of the test points lead to a change of category due to the perturbations.

Hence, it can be concluded that User Opinion has the greatest effect, followed by Consistency, Error Prevention, and Performance having a significant effect, while System Feedback and Error Recovery have a smaller effect on usability.

4) Comparison of the Sensitivity Studies for the Two-FuzzySet and Three-Fuzzy-Set Cases: We note that both the twofuzzy-set and the three-fuzzy-set cases provide a movement in overall usability in the right direction when a particular input factor is perturbed and, therefore, are an appropriate model for determining the overall figure of merit for usability. However, we note that the number of occasions when the three-fuzzy-set case had the movement in the wrong direction (points marked "*") is larger than for the two-fuzzy-set case. This is probably due to the fact that the three-fuzzy-set case has a large number of coefficients that can be adjusted to fit the original data, leading to overfitting of the data and, hence, the generalization capability required when dealing with the perturbed cases is not as good as the two-fuzzy-set cases.

For these reasons, it is adequate to use a two-fuzzy-set model when calculating the overall figure of usability. This is particularly the case, since the computational effort is reduced.

\section{OVERALl Structure of USABILITy EVAluation}

Another very important issue for usability evaluation is to determine precisely when it is to be used. This evaluation could 
TABLE VII

INPUt TEST CASES Derived From Varying Different INPUTS About TWO NOMINAL POINTS OUTPUT TRAINING PAIRS OBTAINED FROM TESTING THE AVERAge INTERfaCe. Three Fuzzy SETS FOR EACH INPUT

\begin{tabular}{l|l|l}
\hline Test Case Number & \multicolumn{1}{|c}{ Input Vector } & \multicolumn{1}{c}{ Usability } \\
\hline 1 & {$[557548]$} & 6.0 nominal pt I \\
\hline 2 & {$[357548]$} & 6.6 \\
\hline 3 & {$[757548]$} & 5.4 \\
\hline 4 & {$[537548]$} & 6 \\
\hline 5 & {$[587548]$} & 5.7 \\
\hline 6 & {$[559548]$} & 6.2 \\
\hline 7 & {$[554548]$} & 6 \\
\hline 8 & {$[557348]$} & 6.1 \\
\hline 9 & {$[557748]$} & $6.2^{*}$ \\
\hline 10 & {$[557528]$} & 6.3 \\
\hline 11 & {$[557568]$} & 5.2 \\
\hline 12 & {$[5575410]$} & 6.8 \\
\hline 13 & {$[557546]$} & 5.6 \\
\hline 14 & {$[457637]$} & 6.1 nominal pt 2 \\
\hline 15 & {$[157637]$} & 6.8 \\
\hline 16 & {$[657637]$} & 5.4 \\
\hline 17 & {$[437637]$} & $5.9^{*}$ \\
\hline 18 & {$[477637]$} & 5.8 \\
\hline 19 & {$[455637]$} & 6.3 \\
\hline 20 & {$[459637]$} & 5.8 \\
\hline 21 & {$[457337]$} & $5.9^{*}$ \\
\hline 22 & {$[457837]$} & 5.1 \\
\hline 23 & {$[457617]$} & 6.5 \\
\hline 24 & {$[457607]$} & 6.7 \\
\hline 25 & {$[457667]$} & 5.1 \\
\hline & &
\end{tabular}

be done at: 1) the design stage; 2) at the stage of an initial prototype, which implements only the UI; and 3) testing on the full software. If they are used with the full implementation of the software, the likelihood of having to make alterations to the software is reduced. However, one can obtain a characterization of the quality of the software from the usability point of view. This could be useful for comparison of different software packages, and for determining its suitability for the given situation. If one were to use the technique at the design stage, then it is more possible to make alterations so that one can obtain an improved design, and eventually an improved UI. The evaluation of an initial prototype system of the UI also permits significant changes to be made to the UI. The new approach to usability measurement proposed in this paper has three components:

1) a heuristic evaluation;

2) a testing aspect;

3) combining the results of these tests, and turning them into a usability measure.

The framework that we employed for developing the usability measure is the fuzzy-systems approach presented in this paper.

If the overall figure of merit generated during the testing and evaluation phase is in the Good range, then one can proceed with developing the full system. If, however, the overall measure of usability is in the Poor or Average range for this particular piece of software, it is necessary to drill down to see, for the measurements taken on this software, which of
TABLE VIII

INPUT TEST CASES DERIVED From VARYING DIFFERENT INPUTS ABOUT Two Nominal POINTS OUTPUt TRAining Pairs ObTAined From Testing the Poor Interface. Three Fuzzy Sets FOR EACH INPUT

\begin{tabular}{|c|c|c|}
\hline Test Case Number & Input Vector & Usability \\
\hline 1 & 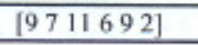 & 1.9 nominal pt 1 \\
\hline 2 & {$\left[\begin{array}{lllllll}6 & 7 & 1 & 1 & 6 & 9 & 2\end{array}\right]$} & 1.9 \\
\hline 3 & {$[107111692]$} & $2.0^{*}$ \\
\hline 4 & {$\left[\begin{array}{lllllll}9 & 5 & 1 & 1 & 6 & 9 & 2\end{array}\right]$} & $1.7^{*}$ \\
\hline 5 & {$\left[\begin{array}{lllllll}9 & 9 & 11 & 6 & 9 & 2\end{array}\right]$} & 1.5 \\
\hline 6 & [978692] & 2 \\
\hline 7 & {$\left[\begin{array}{llll}9 & 7 & 1269 & 9\end{array}\right]$} & 1.8 \\
\hline 8 & {$\left[\begin{array}{lllllll}9 & 7 & 11 & 3 & 9 & 2\end{array}\right]$} & 2.1 \\
\hline 9 & 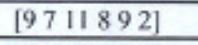 & 1.2 \\
\hline 10 & {$\left[\begin{array}{llllll}9 & 7 & 11 & 6 & 7 & 2\end{array}\right]$} & $1.8^{4}$ \\
\hline 11 & $\{97116102\rceil$ & $2.1^{*}$ \\
\hline 12 & {$[97 \quad 11690]$} & 1.7 \\
\hline 13 & {$\left[\begin{array}{llllll}9 & 7 & 11 & 6 & 9 & 5\end{array}\right]$} & 2 \\
\hline 14 & 17896721 & 2 \\
\hline 15 & {$\left[\begin{array}{llllll}5 & 8 & 9 & 6 & 7 & 2\end{array}\right]$} & 2 \\
\hline 16 & {$\left[\begin{array}{lllllll}9 & 8 & 9 & 6 & 7 & 2\end{array}\right]$} & 1.9 \\
\hline 17 & {$[769672]$} & 2.2 \\
\hline 18 & 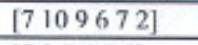 & 1.8 \\
\hline 19 & {$\left[\begin{array}{l}787672] \\
\end{array}\right.$} & 2.1 \\
\hline 20 & 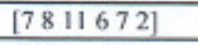 & 1.9 \\
\hline 21 & $\mid 789472\rfloor$ & 2.3 \\
\hline 22 & {$\left[\begin{array}{llllll}7 & 8 & 9 & 8 & 7 & 2\end{array}\right]$} & 1.9 \\
\hline 23 & 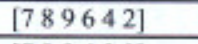 & 2.7 \\
\hline 24 & {$\left[\begin{array}{lllllll}7 & 8 & 9 & 6 & 9 & 2\end{array}\right]$} & 2 \\
\hline
\end{tabular}

the factors appear to be in the range of the Poor subset. This helps identify the particular factors that require further work. The prototype system design and implementation could be reviewed to help improve these particular factors. This iterative process is continued until appropriate usability measures are achieved.

\section{RECAPITULATION}

Both models, namely the ones that use: 1) two fuzzy sets (Good, Poor) and 2) three fuzzy sets (Good, Average, Poor) to span the space for each input factor, successfully capture the relationship between these input factors and the usability value. Further, we note that in both these models, an improvement in the input factor leads to an improvement in the usability value, and a deterioration in the input factor leads to a reduction in the usability value. However, in all cases, we find that the extent of the influence of a particular input factor is also dependent on the score associated with the other input factors. Thus, in some cases, a change in a particular input factor leads to a significant change in the usability value, even causing a change in the category range of the UI. In other cases, it has a much smaller influence. It was only through the use of the fuzzy-system model that we were able to identify the cases that caused a significant change in the usability value. It was also noted that if we only wanted a single overall measure of usability, the two-fuzzy-set representation is adequate. If, however, we wanted to drill down to the individual factors, a three-fuzzy-set model is more appropriate. 
TABLE IX

MEMBERSHIP FUNCTION PARAMETERS (2 MEMBERSHIP FunCtIONS)

\begin{tabular}{l|l|c|c|r}
\hline \multicolumn{1}{c|}{ Input } & Fuzzy Set & $a$ & $b$ & $c$ \\
\hline Feedback & Good & 5.371 & 1.763 & -0.200 \\
\hline & Poor & 5.772 & 1.969 & 10.761 \\
\hline Consistency & Good & 4.814 & 1.998 & -0.181 \\
\hline & Poor & 4.982 & 2.178 & 9.953 \\
\hline Error Prevention & Good & 5.813 & 2.107 & -0.139 \\
\hline & Poor & 5.962 & 2.165 & 11.985 \\
\hline Performance & Good & 4.031 & 2.169 & 0.111 \\
\hline & Poor & 3.723 & 2.063 & 8.234 \\
\hline Error Recovery & Good & 4.930 & 1.971 & -0.089 \\
\hline & Poor & 5.127 & 2.000 & 9.884 \\
\hline User Opinion $\dagger$ & Poor & 5.052 & 2.152 & 1.106 \\
\hline & Good & 4.865 & 1.936 & 11.151 \\
\hline
\end{tabular}

TABLE $X$

Membership Function PARAMETERS (3 MEMbership FunCtions)

\begin{tabular}{l|l|c|r|r}
\hline \multicolumn{1}{c|}{ Input } & Fuzzy Set & $a$ & $b$ & \multicolumn{1}{c}{$c$} \\
\hline Feedback & Good & 3.048 & 1.704 & 0.165 \\
\hline & Average & 2.677 & 1.947 & 5.534 \\
\hline & Poor & 2.736 & 2.02 & 10.998 \\
\hline Consistency & Good & 2.676 & 1.972 & 0.152 \\
\hline & Average & 2.408 & 1.726 & 4.924 \\
\hline & Poor & 2.524 & 1.984 & 9.983 \\
\hline Error Prevention & Good & 3.145 & 2.031 & 0.132 \\
\hline & Average & 3.233 & 1.907 & 6.064 \\
\hline & Poor & 3.012 & 1.995 & 11.99 \\
\hline Performance & Good & 2.249 & 1.906 & 0.167 \\
\hline & Average & 1.959 & 1.966 & 3.891 \\
\hline & Poor & 2.099 & 2.004 & 7.896 \\
\hline Error Recovery & Good & 2.467 & 2.007 & -0.021 \\
\hline & Average & 2.472 & 1.979 & 5.056 \\
\hline & Poor & 2.435 & 2.182 & 9.947 \\
\hline & Poor & 2.508 & 1.996 & 1.006 \\
\hline & Average & 2.533 & 2.018 & 5.888 \\
\hline & Good & 2.704 & 1.885 & 10.87 \\
\hline & & & &
\end{tabular}

\section{APPENDIX}

Tables IX and $\mathrm{X}$ give the parameters obtained for the membership functions for the two- and three-fuzzy-set-per-inputfactor cases.

\section{REFERENCES}

[1] R. F. Bateman, A Translator to Encourage User Modifiable Machine Dialogue. Great Malvern, U.K.: Royal Signals and Radar Establishment. 1983.

[2] N. Bevan and M. Macleod, "Usability measurement in context," Behav. Inf. Technol., vol. 13, no, 1/2, pp. 132-145, 1994.

[3] E. Chang, "Object-oriented user interface design and usability evaluation," M.S. thesis, Dept. Comput. Sci. Comput. Eng., La Trobe Univ., Melbourne, Vic., Australia. 1996.

[4] E. Chang and T. Dillon, "Automatic usability testing in human computer interactions," in INTERACT 97. S. Howard. J. Hammond, and G. Lingaard, Eds. London: Chapman and Hall, 1997, pp. 77-84.

[5] A. Dillon and M. Maquire, "Usability measurement-Its practical value to the computer industry," in Proc. ACM/FIP Human Factors Computer System (INTERCHI), Amsterdam, The Netherlands, 1993, pp. 145-148.

[6] B. R. Gaines, "The technology of interaction-dialogue programming rules," Int. J. Man-Mach. Stud., vol. 14, no. I, pp. 133-150, 1981.

[7] J. Levy and J. Nielsen, "Measuring usability preference vs, performance," Commun. ACM, vol. 37, no. 4, pp. 66-75, Apr. 1994.

[8] T. G. Lewis, F. Handloser, III, S. Bose, and S. Yang. "Prototypes from standard user interface management systems," IEEE Trans. Comput. vol. 22, no. 5, pp. 51-60, May 1989.
[9] E. H. Mamdani and S. Assilian. "An experiment in linguistic synthesis with a fuzzy logic controller," Int. J. Man-Mach. Stud., vol. 7, no. I, pp. 1-13, 1975.

[10] R. Molich and J. Nielsen, "Heuristic evaluation of user interfaces," in Proc. ACM Human Factors Computing Systems (CHI). New York, 1990, pp. 249-256.

[11] T. P. Moran, "The command language grammar: A representation for the user interface of interactive systems," Int. J. Man-Mach. Stud., vol. 15, no. 1, pp. 3-50, 1981.

[12] J. Nielsen and V. Philips, "Estimating the relative usability of two interfaces: Heuristic, formal, and empirical methods compared," in Proc. ACM/IFIP Human Factors Computing Systems (INTERCHI), Amsterdam. The Netherlands. 1993, pp. 214-221.

[13] J. Nielsen, "Enhancing the explanatory power of usability heuristics," in Proc. ACM Human Factors Computing Systems (CHI), Boston, MA. 1993, pp. 152-158.

[14] S. Sestito and T. Dillon, Automated Knowledge Acquistion. Sydney, Australia: Prentice-Hall. 1993.

[15] B. Shackel, "The concept of usability," in Proc. IBM Software and Information Usability Symp., Poughkeepsie, NY, Sep. 1981, pp. 1-30.

[16] T. Takagi and M. Sugeno, "Fuzzy identification of systems and its applications to modelling and control," IEEE Trans. Syst., Man, Cybern., vol. 15. SMC-IS, no, 1, pp. 116-131, 1985.

[17] M. J. Tauber, "Top down design of human-computer interfaces," in Visual Languages, Management and Information System. New York: Plenum, 1986, pp. 393-429.

[18] H. Thimbleby, "Formulating usability," SIGCHI Bull., vol. 26, no. 2 , pp. 59-64, Apr. 1994.

[19] S. Treu, User Interface Evaluation-A Structured Approach. New York: Plenum, 1994, pp. 175-195.

[20] R. J. S. Jang, "ANFIS: Adaptive-network-based fuzzy inference system," IEEE Trans. Syst., Man, Cybern., vol. 23, no. 3, pp. 665-685, May-Jun. 1993.

[21] E. Chang. T. S. Dillon, and D. Cook, "Measurement of usability of software using a fuzzy system approach," in Invited Key Note Paper Proc. 8th Int. Conf. Software Engineering and Knowledge Engineering, Lake Tahoe, NV, 1996, pp. 69-76.

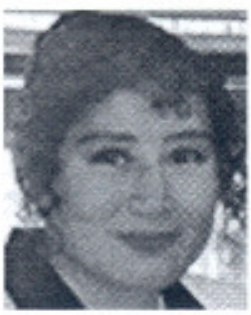

Elizabeth Chang ( $\left.\mathrm{M}^{*} 93\right)$ is the Professor of Software Engineering in Curtin Business School (CBS), Curtin University of Technology, Perth, W.A. Australia, Director of Frontier Technology for Extended Enterprise Center at CBS, and Founder of the Center for IT Applications and Logistics Infor. matics at Newcastle University, Callaghan, N.S.W. Australia. She has written over 100 papers in international and national journals and conferences, and has authored one book. Her research interests include user-interface analysis and design, usability evaluation. plug-and-play component-based system, Internet computing, including $\mathrm{XML}$ systems, trust and reputation, and ontologies.

She is a Member of the ACM and the Australian Computer Society.

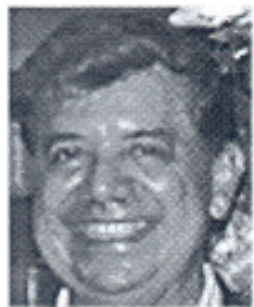

Tharam S. Dillon (M'74-SM' $87-F^{\prime} 98$ ) is the Dean of the Faculty of Information Technology at University of Technology Sydney (UTS), Sydney, N.S.W. Australia. He has also worked with industry and commerce in developing systems in telecommunications, health care systems, e-commerce, logistics, power systems, and banking and finance. $\mathrm{He}$ is Editor-in-Chief of the International Journal of Computer Systems Science and Engineering and the International Journal of Engineering Intelligent Systems, as well as coeditor of the Journal of Electric Power and Energy Systems. He is on the advisory editorial board of Applied Intelligence, published by Kluwer in the U.S., and Computer Communications. published by Elsevier in the U.K. He has published more than 400 papers in international and national joumals and conferences, and has written four books and edited five other books. His research interests include data mining. Internet computing, e-commerce, hybrid neurosymbolic systems, neural nets, software engineering, database systems, and computer networks.

$\mathrm{He}$ is a Fellow of the Institution of Engineers (Australia) and of the Australian Computer Society. 